\title{
Structural Integrity of the Alveolar-Capillary Barrier in Cynomolgus Monkeys Challenged with Fully Virulent and Toxin-Deficient Strains of Bacillus anthracis
}

Felice D'Agnillo, Xiaoyuan Zhang, and Matthew C. Williams

From the Laboratory of Biochemistry and Vascular Biology, Office of Blood Research and Review, Center for Biologics Evaluation and Research (CBER), US Food and Drug Administration (FDA), Silver Spring, Maryland

\author{
Accepted for publication \\ June 9, 2020. \\ Address correspondence to \\ Felice D'Agnillo, Ph.D., Center \\ for Biologics Evaluation and \\ Research, US Food and Drug \\ Administration Federal \\ Research Center at White Oak, \\ Building 52/72, Room 4110, \\ 10903 New Hampshire Ave. \\ Silver Spring, MD 20993- \\ 0002. E-mail: felice.dagnillo@ \\ fda.hhs.gov.
}

\begin{abstract}
Inhalational anthrax, a disease caused by inhaling Bacillus anthracis spores, leads to respiratory distress, vascular leakage, high-level bacteremia, and often death within days. Anthrax lethal toxin and edema toxin, which are composed of protective antigen (PA) plus either lethal factor (LF) or edema factor (EF), respectively, play an important yet incompletely defined role in the pulmonary pathophysiology. To better understand their contribution, we examined the structural integrity of the alveolar-capillary barrier in archival formalin-fixed lungs of cynomolgus monkeys challenged with the fully virulent $B$. anthracis Ames wild-type strain or the isogenic toxin-deficient mutants $\Delta \mathrm{EF}, \Delta \mathrm{LF}$, and $\triangle \mathrm{PA}$. Pulmonary spore challenge with the wild-type strain caused high mortality, intra-alveolar hemorrhages, extensive alveolar septal sequestration of bacteria and neutrophils, diffuse destabilization of epithelial and endothelial junctions, increased markers of coagulation and complement activation (including tissue factor and $\mathrm{C} 5 \mathrm{a}$ ), and multifocal intra-alveolar fibrin deposition. $\Delta \mathrm{EF}$ challenge was lethal and showed similar alveolar-capillary alterations; however, intra-alveolar hemorrhages, bacterial deposition, and markers of coagulation or complement were absent or markedly lower. In contrast, $\Delta \mathrm{LF}$ or $\triangle \mathrm{PA}$ challenges were nonlethal and showed no signs of alveolar bacterial deposition or alveolar-capillary changes. These findings provide evidence that lethal toxin plays a determinative role in bacterial dissemination and alveolar-capillary barrier dysfunction, and edema toxin may significantly exacerbate pulmonary pathologies in a systemic infection. (Am J Pathol 2020, 190: 2095-2110; https://doi.org/10.1016/j.ajpath.2020.06.007)
\end{abstract}

Inhalational anthrax is a disease caused by inhaling spores of the Gram-positive bacterium Bacillus anthracis. Inhaled spores are transported to regional lymph nodes by lung phagocytic cells where spore germination and proliferation lead to the release of vegetative bacilli into the systemic circulation. ${ }^{1,2}$ Fully virulent strains of $B$. anthracis carry two plasmids, $\mathrm{pXO} 1$ and $\mathrm{pXO} 2$, which contain the genes to synthesize anthrax toxin and the antiphagocytic poly- $\gamma-\mathrm{D}-$ glutamic acid capsule, respectively. Anthrax toxin, a key virulence factor of $B$. anthracis, plays a multifaceted role during the early and late stages of anthrax infection. ${ }^{1-3}$

Anthrax toxin consists of three proteins: protective antigen (PA), lethal factor (LF), and edema factor (EF). PA combines with LF to form lethal toxin (LT), whereas PA and EF together form edema toxin (ET). ${ }^{1-3}$ PA shuttles LF and EF into cells by receptor-mediated endocytosis involving at least two identified cell surface anthrax toxin receptors, capillary morphogenesis protein 2 and tumor endothelial marker 8 . LF is a protease that inactivates mitogen-activated protein kinase signaling by cleaving all the upstream mitogen-activated protein kinase kinases

\footnotetext{
Supported by US Food and Drug Administration (FDA) Modernizing Science funds (F.D.).

X.Z. and M.C.W. contributed equally to this work.

Disclosures: None declared.
} 
(except mitogen-activated protein kinase kinase 5). EF acts as a calcium/calmodulin-dependent adenylate cyclase that significantly increases intracellular cAMP production. ${ }^{1-4}$ Historically, LT has generally been thought to play a more critical pathogenic role than ET, although the contributory actions of the latter have been increasingly recognized. $^{5-10}$ A common feature of both LT and ET seems to be their ability to impair innate and adaptive immune responses. ${ }^{1,2,11-14}$

An untreated systemic anthrax infection is usually fatal, with high mortality rates persisting even after aggressive antimicrobial therapy and supportive care. Hallmark features of systemic anthrax disease include high-level bacteremia, vascular leakage, pleural effusion, respiratory distress, hemorrhages, and vasculitis. ${ }^{15-18}$ Progressive and persistent pleural effusions and hemorrhages were significant findings in the inhalational anthrax patients of the 2001 bioterrorism attack in the United States. ${ }^{15}$ Pulmonary hemorrhages, disseminated intravascular coagulation, and vasculitis were prominent observations in the inhalational anthrax victims of the 1979 Sverdlovsk incident. ${ }^{16}$ The nature of these pulmonary pathologies suggests that the alveolar-capillary barrier may be a prime pathogenic target of systemic anthrax disease.

The alveolar-capillary barrier plays a central role in gas exchange, permeability regulation, fluid clearance, and host defense. ${ }^{19,20}$ This barrier consists of: i) the alveolar epithelium composed of squamous type 1 (AT1) and cuboidal type 2 cells (AT2), ii) the alveolar capillary endothelium, and iii) the alveolar basement membrane formed by a network of collagen type IV (Col IV) and laminin that separates the epithelium and endothelium and stabilizes the alveolar architecture. Permeability across the alveolar epithelium and endothelium is tightly regulated by intercellular junctional complexes, including cadherin-expressing adherens junctions and claudin-rich tight junctions. ${ }^{21,22}$ Damage to epithelial or endothelial junctions results in barrier dysfunction, which is often a key feature of acute lung injury, acute respiratory distress syndrome, and microbial infections. ${ }^{21-23}$ Previous cell culture studies have reported that anthrax toxin directly impairs pulmonary epithelial and endothelial barrier function by altering cell contractility and destabilizing intercellular junctional complexes. ${ }^{24-29}$ Despite these extensive in vitro observations, relatively little is known about how the actions of anthrax toxin modulate alveolar-capillary barrier integrity in the setting of an actual anthrax infection.

Mortality, edema, and vascular collapse are observed in animals treated with purified LT or ET. ${ }^{1,6-9,18}$ Toxin challenge models, however, do not fully reproduce the pathophysiologic features of systemic anthrax disease, which underscores the importance of studying the contributions of LT and ET in a spore challenge model. ${ }^{1,18,30}$ Nonhuman primates are considered highly relevant and useful models of human inhalational anthrax because these species exhibit similar pulmonary pathologies and disease progression after exposure to aerosolized B. anthracis spores. ${ }^{18,31-34}$ In a previous study, pulmonary spore challenge in cynomolgus macaques using the fully virulent $B$. anthracis Ames strain and isogenic toxin deletion mutants showed that LT, but not ET, was required to induce extensive bacteremia and death. $^{34}$ These prior observations were spore dose dependent, and general histopathologic features in the lung, liver, spleen, and brain were consistent with those described in clinical cases and other nonhuman primate models. ${ }^{15-18}$ Here, using archival formalin-fixed lung specimens from this previous study, ${ }^{34}$ we qualitatively and semiquantitatively evaluated the effects of these different spore challenges on the structural integrity of the alveolar-capillary barrier. We found that infections with LT-producing strains produced structural disruption of the alveolar-capillary barrier and related pathologies that were, in some cases, aggravated by ET.

\section{Materials and Methods}

\section{Archival Lung Samples from Cynomolgus Macaques}

This retrospective study was performed by using archival formalin-fixed, paraffin-embedded (FFPE) lung specimens from spore challenge cynomolgus macaque experiments conducted at the Lovelace Respiratory Research Institute (LRRI, Albuquerque, NM). ${ }^{34}$ All animal procedures were performed in accordance with the guidelines outlined in the Guide for the Care and Use of Laboratory Animals ${ }^{35}$ and approved by the Institutional Animal Care and Use Committee at the LRRI. Vietnamese cynomolgus macaques were challenged by bronchoscopy with spores of the fully virulent Ames strain of $B$. anthracis (WT) that produces both LT and ET or isogenic toxin-deficient mutants lacking EF $(\Delta \mathrm{EF}), \mathrm{LF}(\Delta \mathrm{LF})$, or PA $(\Delta \mathrm{PA})$ generated from the Ames parental strain by replacing the cya, lef, and pagA coding sequences, respectively. Cultures of the WT and mutant strains exhibited similar growth curves. Lung specimens were obtained from 12 monkeys challenged at the following spore doses (colony-forming units): WT, $5.8 \times 10^{7} ; \Delta \mathrm{EF}$ or $\Delta \mathrm{LF}, 5.7 \times 10^{7}$; and $\Delta \mathrm{PA}, 9.1 \times 10^{7}(n=3$ animals per challenge group). All three monkeys in the WT or $\Delta \mathrm{EF}$ groups were moribund or died within 2 to 3 days or 3 to 5 days, respectively. All $\Delta \mathrm{LF}$ and $\Delta \mathrm{PA}$ animals survived the challenge and were euthanized at 21 days $(\Delta \mathrm{LF})$ or 14 days $(\Delta \mathrm{PA})$. Animals at study termination or considered moribund during the clinical observation period were euthanized by using sodium pentobarbital. Harvested lungs infused with $10 \%$ neutral-buffered formalin were fixed, trimmed, and processed.

\section{Lung Histology}

FFPE lung sections 5- $\mu \mathrm{m}$ thick were dewaxed, rehydrated, and stained by using hematoxylin and eosin for general histology and Brown and Brenn for bacterial detection. Hematoxylin and eosin-stained sections were evaluated for 
focal thickening of the alveolar membrane, congestion, pulmonary edema, alveolar wall cellularity, intra-alveolar hemorrhage, and interstitial and intra-alveolar leukocyte infiltration. Semi-quantitative analyses of intra-alveolar hemorrhages and bacterial dissemination were performed in a blinded fashion (F.D., X.Z., M.C.W.) on highresolution digital images of entire slides captured by using a Hamamatsu NanoZoomer 2.0-RS whole-slide digital scanner and processed with NDP.view2 imaging software version 2.7.52 (Hamamatsu Photonics, Hamamatsu City, Japan). The whole slide digital image of each pulmonary section was divided into 10 to 15 equal-sized areas $\left(16 \mathrm{~mm}^{2}\right.$ each). For intra-alveolar hemorrhages, each field was scored by using the following scale (0-4): 0 , no detectable hemorrhages; 1 , detectable (alveoli with $\leq 10$ intra-alveolar red blood cells present in $<10 \%$ of the stained area); 2 , low (alveoli with $\leq 10$ intra-alveolar red blood cells present in $10 \%$ to $25 \%$ of the stained area); 3 , high (marked hemorrhages with $>10$ intra-alveolar red blood cells per alveoli present in multiple alveoli covering $>25 \%$ of the stained area but intact alveolar septa); and 4, severe (multiple alveoli with marked hemorrhages and three or more areas with damaged alveolar septa). The individual field scores ( $n=10-15$ scores per animal section) were averaged, and a final score was derived ( $n=3$ animals per group). For bacterial deposition, each field was scored by using the following scale (0-4): 0 , no detectable bacteria; or $1,2,3$, and 4 , bacteria observed within $<10 \%, 10 \%$ to $25 \%, 25 \%$ to $50 \%$, and $>50 \%$ of the alveolar septal segments within the field, respectively. The individual field scores $(n=10$ to 15 scores per animal section) were averaged, and a final score was derived ( $n=3$ animals per group).

\section{Antibodies}

Antibodies to E-cadherin (E-cad, \#ab40772), neutrophil elastase (NE, \#ab68672), myeloperoxidase (MPO, \#ab9535), Col IV (\#ab6586), complement component 9 (C9, \#ab173302), and lytic membrane attack complex C5b9 (\#ab55811) were purchased from Abcam (Cambridge, MA). Mouse monoclonal antibodies to claudin-5 (\#35-2500), tissue factor clone TF9-10H10 (TF, \#MA1-83495), complement C5a/C5a des-Arg clone 2952 (C5a, \#MA1-40162), and the Alexa Fluor-conjugated antibodies were purchased from Thermo Fisher Scientific (Waltham, MA). Mouse monoclonal antibody to ETS-related gene clone 9FY (ERG, \#CM421) was obtained from Biocare Medical (Pacheco, CA). A mouse monoclonal antibody that specifically recognizes fibrin (not fibrinogen) (clone 59D8, \#MABS2155) was purchased from EMD Millipore (Burlington, MA).

\section{Immunohistochemistry}

FFPE lung sections 3- $\mu \mathrm{m}$ thick were dewaxed, rehydrated, and heat-treated in a microwave oven for 15 minutes in 10 $\mathrm{mmol} / \mathrm{L}$ sodium citrate buffer $(\mathrm{pH} 6.0)$ or $10 \mathrm{mmol} / \mathrm{L}$ Tris/1
mmol/L EDTA buffer ( $\mathrm{pH}$ 9.0). After cooling for $30 \mathrm{mi}-$ nutes at room temperature, slides were washed in phosphate-buffered saline with $0.05 \%$ Tween-20 and incubated with Bloxall (Vector Laboratories, Burlingame, CA) for 15 minutes at room temperature to inhibit endogenous peroxidases. After a brief wash, sections were blocked in $2.5 \%$ horse serum for 30 minutes at room temperature and incubated overnight at $4{ }^{\circ} \mathrm{C}$ with antibodies to E-cad, claudin-5, ERG, NE, MPO, or TF in phosphate-buffered saline with $0.05 \%$ Tween- 20 containing $2.5 \%$ horse serum. For negative controls, sections were incubated without the primary antibody. Signal was developed by using polymeric peroxidase-conjugated secondary antibodies (ImmPRESS Kit) and ImmPACT DAB (Vector Laboratories). Slides were then washed, dehydrated in graded ethanol and SafeClear II (xylene substitute), and mounted using Permount (Thermo Fisher Scientific). Images were captured by using an Olympus IX71 inverted microscope fitted with Plan Apochromat $40 \times, 60 \times$, and $100 \times$ objectives and an Olympus DP80 digital camera and processed by using cellSens imaging software version 1.12 (Olympus Life Science, Tokyo, Japan). High-resolution digital images of entire slides were also captured by using a Hamamatsu NanoZoomer 2.0-RS whole-slide digital scanner and processed with NDP.view2 imaging software.

\section{Immunohistochemistry Image Analysis}

For semi-quantitative and blinded evaluation of E-cad and claudin-5 staining, a total of 20 to 25 high-resolution images from randomly selected areas per lung section were captured at $400 \times$ magnification, and each image was divided into four quadrants. Alveolar septal E-cad and claudin-5 staining intensity and pattern were scored for each quadrant using the following scale (0-4): 0 , absent; 1 , weak with no linearly concentrated staining; 2, moderate with limited linear staining; 3, strong with well-defined linearly organized expression; and 4, intense immunoreactivity with prominent, linearly organized expression present throughout quadrant. The quadrant scores were averaged for each image, and the total image scores $(n=20$ to 25 per animal section) were averaged to derive a final score $(n=3$ animals per group).

For endothelial ERG image analyses, a total of 20 highresolution images from randomly selected fields per section were acquired at $400 \times$ magnification. For each image, the total number of ERG-positive nuclei were counted and averaged ( $n=20$ images per section), and a final total count was derived ( $n=3$ animals per group). For ERG staining intensity, 50 to 80 positive cells per image ( $n=7$ images per section) were individually outlined, and a gray value per nucleus was measured by using ImageJ software version 1.46r (NIH, Bethesda, MD; http://imagej.nih.gov/ij). The nuclei were further defined as low, moderate, and high expression based on preassigned gray value ranges, and the total percentage for each category was calculated as a 
function of the total nuclei analyzed per image. The values for each image per section were averaged, and a final value was derived ( $n=3$ animals per group).

For NE and MPO image analyses, a total of 20 images from randomly selected fields per section were acquired at $400 \times$ magnification. For each image, the total number of NE- or MPO-positive cells was counted, averaged, and a final count derived ( $n=3$ animals per group).

\section{Immunofluorescence Staining and Image Analysis}

FFPE slides 3- $\mu \mathrm{m}$ thick were dewaxed, rehydrated, and heat-treated in a microwave oven for 15 minutes in 10 $\mathrm{mmol} / \mathrm{L}$ sodium citrate buffer $(\mathrm{pH}$ 6.0) or $10 \mathrm{mmol} / \mathrm{L}$ Tris/1 mmol/L EDTA buffer ( $\mathrm{pH} 9.0$ ), and cooled for 30 minutes at room temperature. Sections were then blocked in phosphate-buffered saline with $0.05 \%$ Tween- 20 with $2.5 \%$ bovine serum albumin for 30 minutes at room temperature followed by overnight incubation at $4{ }^{\circ} \mathrm{C}$ with antibodies to E-cad, claudin-5, ERG, Col IV, TF, C5a, MPO, C9, C5b9, or fibrin. Sections were rinsed and incubated with Alexa Fluor 488- and Alexa Fluor 647-conjugated secondary antibodies for 1 hour at room temperature. Nuclei were counterstained with Hoechst 33342. For double-labeling experiments, primary antibodies were mixed together and incubated overnight at $4^{\circ} \mathrm{C}$. Sections stained with secondary antibodies alone showed no specific staining. Images were captured by using an Axio Observer Z1 inverted microscope equipped with an Axiocam 506 monochrome camera, an ApoTome. 2 optical sectioning system, and a PlanApochromat 63x/1.4NA oil immersion objective lens (Carl Zeiss, Thornwood, NY). Digital image postprocessing and analysis were performed by using the ZEN 2 version 2.0 imaging software. Whole slide fluorescence imaging was also performed by using a Hamamatsu NanoZoomer 2.0-RS whole-slide digital scanner with a fluorescence module \#L11600, and NDP.view2 software was used for processing.

\section{Statistical Analysis}

Data are expressed as means \pm SEM. Statistical comparisons were performed by a one-way analysis of variance for planned comparisons and post hoc Bonferroni's test for multiple comparisons between equal-sized groups (Prism 5 software, GraphPad Software, La Jolla, CA). In all analyses, $P<0.05$ was taken as the level of statistical significance.

\section{Results}

Pulmonary Histology, Intra-alveolar Hemorrhages, and Bacterial Dissemination

Archival FFPE terminal lung sections were obtained from monkeys challenged with spores of the fully virulent $B$. anthracis Ames strain (WT) that produces both LT and ET or the isogenic mutants $\triangle \mathrm{EF}, \triangle \mathrm{LF}$, and $\triangle \mathrm{PA}$. The animal cohort examined in this retrospective study showed $100 \%$ lethality with WT (median time of death, 54 hours) or $\Delta \mathrm{EF}$ (median time of death, 80 hours) compared with $100 \%$ survival with $\Delta \mathrm{LF}$ or $\Delta \mathrm{PA}\left(n=3\right.$ monkeys per group). ${ }^{34}$ Hematoxylin and eosin staining revealed clear histologic differences between WT lungs and either $\Delta$ LF or $\Delta$ PA lungs (Figure 1, A, G, and J). These findings included marked and diffuse intra-alveolar hemorrhages, alveolar wall thickening, diffuse alveolar interstitial and capillary infiltrates with minimal intra-alveolar localization, increased septal red blood cell congestion, multifocal intra-alveolar hyaline membranes, and focal to multifocal intra-alveolar epithelial sloughing with proteinaceous debris of varying intensity (Figure 1A). Compared with WT lungs, $\Delta \mathrm{EF}$ lungs exhibited less diffuse alveolar wall thickening and congestion, fewer intra-alveolar hyaline membranes and debris, and minimal or variably detectable small focal intra-alveolar hemorrhages (Figure 1D). Brown and Brenn staining identified extensive and widely disseminated bacterial deposition in WT lungs (Figure 1, B and C). Compared with WT lungs, $\Delta \mathrm{EF}$ lungs showed less diffuse deposition and shorter chain bacterial aggregates (Figure 1, E and F). Bacilli were not detectable in $\Delta \mathrm{LF}$ or $\Delta \mathrm{PA}$ lungs (Figure $1, \mathrm{H}$ and $\mathrm{K}$ ). In both WT and $\Delta \mathrm{EF}$ lungs, bacilli aggregates were primarily sequestered in alveolar septal capillaries and interstitium with minimal intra-alveolar distribution (Figure 1, C and F). Whole-slide image analyses further underscored the significant degree of intra-alveolar hemorrhages and bacterial deposition in WT lungs relative to the other three groups (Figure 1, I and L).

\section{Reduced E-Cad in the Alveolar Epithelium after WT and $\Delta \mathrm{EF}$ Challenge}

To assess intercellular junctional integrity of the alveolar epithelium, the expression of E-cad, the major cadherin in epithelial adherens junctions, was examined by immunohistochemistry and immunofluorescence. In $\Delta \mathrm{LF}$ and $\triangle \mathrm{PA}$ lungs, E-cad immunoreactivity was highly concentrated along the lateral borders of alveolar epithelial type 1 cells, with particularly high expression at intercellular contacts with alveolar epithelial type 2 cells (Figure 2, I, J, M, and $\mathrm{N})$. Compared with $\triangle \mathrm{LF}$ and $\triangle \mathrm{PA}$, WT and $\Delta \mathrm{EF}$ showed a diffuse pattern of reduced and discontinuous E-cad staining (Figure 2, A, B, E, and F). In WT lungs, a near-complete absence of E-cad signal was noted in the epithelium of severely injured and hemorrhagic alveoli (Figure 2, A and B). Semi-quantitative image analyses of staining intensity and distribution further revealed the greater E-cad expression in $\triangle \mathrm{LF}$ and $\triangle \mathrm{PA}$ relative to $\mathrm{WT}$ and $\triangle \mathrm{EF}$ (Figure $2 \mathrm{O}$ ). Representative immunofluorescence imaging combined with differential interference contrast further identified robust E-cad localization along epithelial borders in $\triangle \mathrm{PA}$ lungs (Figure 2, C and D), whereas WT lungs exhibited 


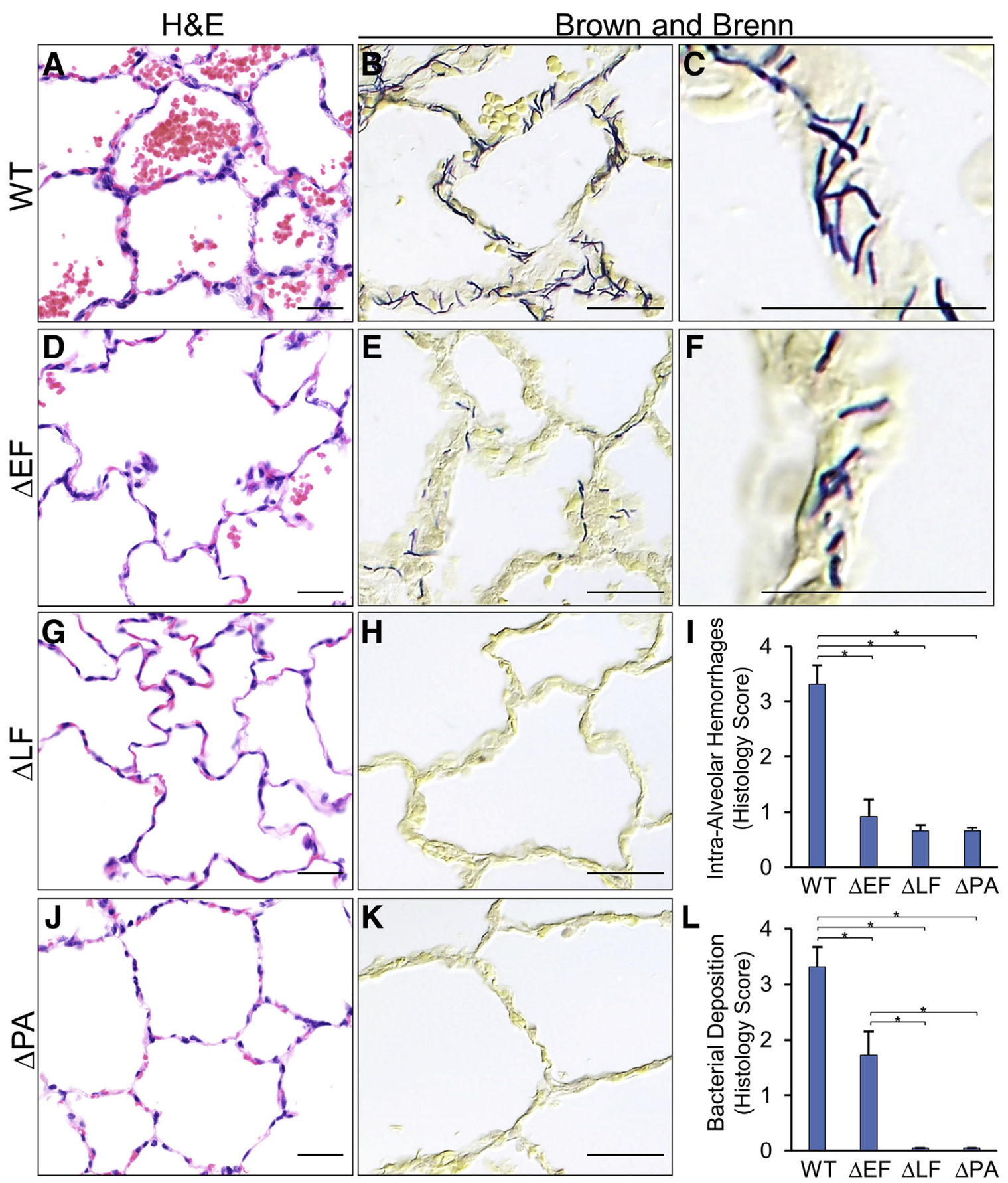

Figure 1 Lung histology, intra-alveolar hemorrhages, and bacteria deposition. Representative images of hematoxylin and eosin (H\&E) and Brown and Brenn staining of terminal monkey lung sections following bronchoscopic spore challenge with Bacillus anthracis Ames strain (wild-type [WT]) (A-C) or deletion mutant strains lacking edema factor $(\Delta \mathrm{EF})(\mathbf{D}-\mathbf{F})$, lethal factor $(\Delta \mathrm{LF})(\mathbf{G}$ and $\mathbf{H})$, or protective antigen $(\Delta \mathrm{PA})(\mathbf{J}$ and $\mathbf{K})$. WT shows intra-alveolar hemorrhages and bacterial dissemination (dark blue, rod-shaped) within alveolar septa. Lower but detectable hemorrhages and bacteria are observed with $\Delta \mathrm{EF}$ but not with $\Delta \mathrm{LF}$ or $\triangle \mathrm{PA}$. I and L: Semi-quantitative analyses of intra-alveolar hemorrhages and bacterial deposition in alveolar septa. For each parameter, the score values are reported as the means \pm SEM for three animals per challenge group. ${ }^{*} P<0.05$. Scale bars: $20 \mu \mathrm{m}(\mathbf{A}-\mathbf{H}, \mathbf{J}$, and $\mathbf{K})$. Original magnification: $\times 400(\mathbf{A}, \mathbf{B}, \mathbf{D}, \mathbf{E}, \mathbf{G}, \mathbf{H}, \mathbf{J}$, and $\mathbf{K}) ; \times 600(\mathbf{C}$ and $\mathbf{F})$.

diminished junctional staining in both alveolar epithelial type 1 and type 2 cells (Figure 2, G and H). The E-cad antibody was confirmed to be specific for epithelial junctions and did not cross-react with endothelium (Figure 2, $\mathrm{K}$ and $\mathrm{L}$ ).

\section{Reduced Alveolar Capillary Claudin-5 and ERG Expression after WT and $\Delta$ EF Challenge}

To investigate the junctional integrity of alveolar capillary endothelium, we examined the expression of claudin-5, the 

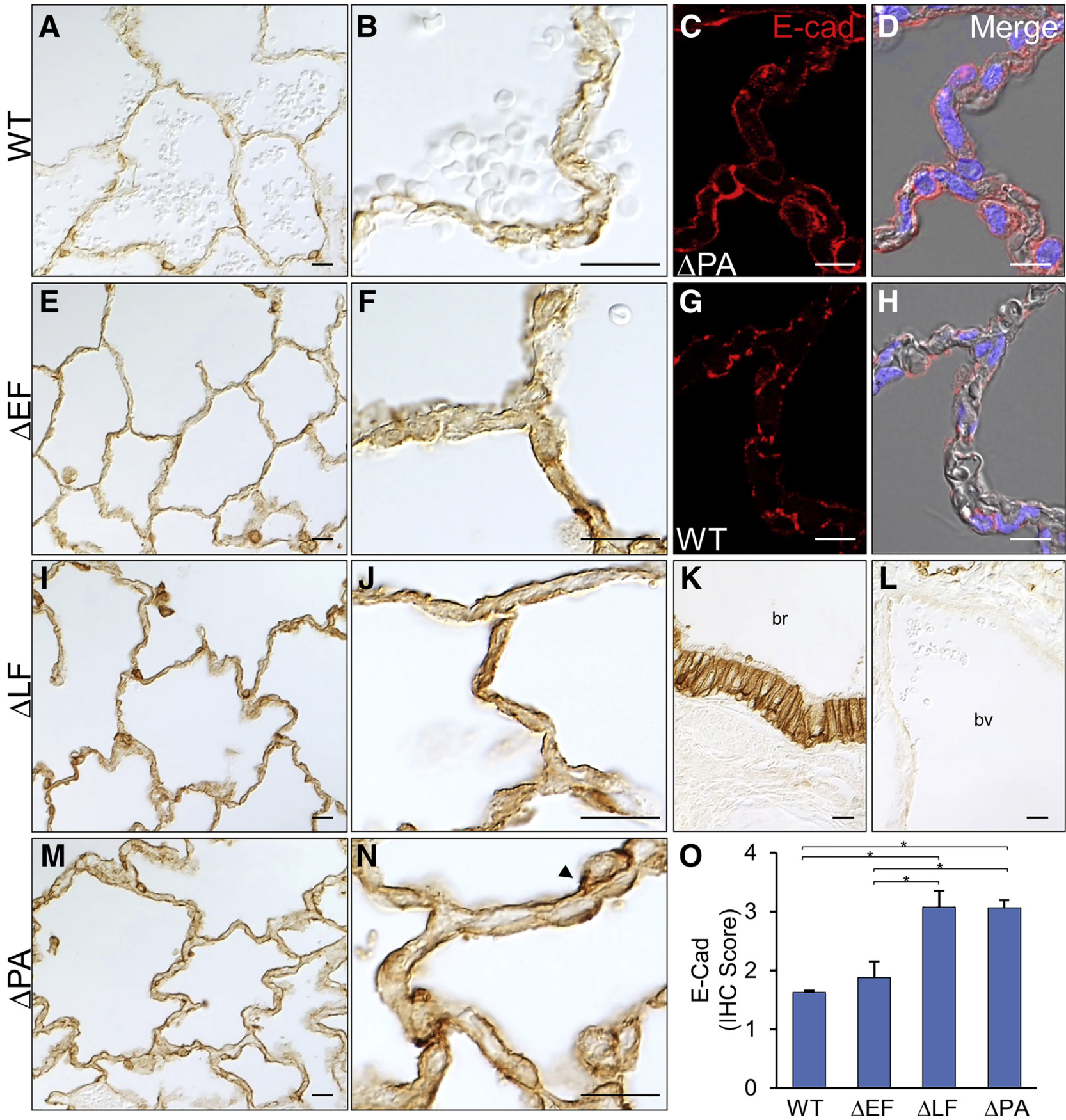

Figure 2 E-cadherin (E-cad) expression in the alveolar epithelium. Representative immunohistochemical images of alveolar epithelial E-cad expression following spore challenge Bacillus anthracis Ames strain [with wild-type (WT)] (A and B), deletion mutant strains lacking edema factor ( $\Delta \mathrm{EF})$ (E and $\mathbf{F})$, lethal factor $(\Delta \mathrm{LF})(\mathbf{I}$ and $\mathbf{J})$, or protective antigen $(\Delta \mathrm{PA})(\mathrm{M}$ and $\mathbf{N})$. For $\Delta \mathrm{LF}$ and $\Delta \mathrm{PA}$, E-cad immunoreactivity (diaminobenzidine staining, brown) is concentrated linearly along alveolar epithelial type 1 borders with particularly high basolateral expression in alveolar epithelial type 2 cells (N, arrowhead). WT and $\Delta$ EF show reduced and discontinuous E-cad staining (A, B, E, and F). Representative E-cad (red) immunofluorescence images merged with differential interference contrast of $\triangle \mathrm{PA}$ (C and $\mathbf{D}$ ) and WT (G and $\mathbf{H}$ ) lungs. Nuclei were counterstained with Hoechst 33342 (blue). Representative images of bronchial epithelium (K) and a large blood vessel (L) demonstrate the specificity of the E-cad antibody for epithelial junctions. Semi-quantitative analysis of E-cad immunoreactivity is shown (0). Score values are reported as the means \pm SEM for three animals per challenge group. ${ }^{\star} P<0.05$. Scale bars: $10 \mu \mathrm{m}(\mathbf{A}-\mathbf{N})$. Original magnification: $\times 400(\mathbf{A}, \mathbf{E}, \mathbf{I}, \mathbf{M}, \mathbf{K}$, and L); $\times 1000(\mathbf{B}, \mathbf{F}, \mathbf{J}$, and N); $\times 600(\mathbf{C}, \mathbf{D}, \mathbf{G}$, and H). br, bronchiole lumen; bv, blood vessel lumen; IHC, immunohistochemistry.

main transmembrane claudin member of endothelial tight junctions. $\triangle \mathrm{LF}$ and $\triangle \mathrm{PA}$ lungs showed robust claudin-5 expression along the borders of alveolar capillary loops (Figure 3, G, H, J and K). In contrast, WT and $\Delta$ EF exhibited a diffuse pattern of diminished and irregular claudin-5 immunoreactivity (Figure 3, A, B, D, and E). Semiquantitative image analyses of staining intensity and distribution further corroborated the higher claudin-5 expression in $\Delta \mathrm{LF}$ and $\Delta \mathrm{PA}$ relative to $\mathrm{WT}$ and $\Delta \mathrm{EF}$ (Figure $3 \mathrm{I}$ ). To further verify endothelial localization of claudin-5, lung sections 

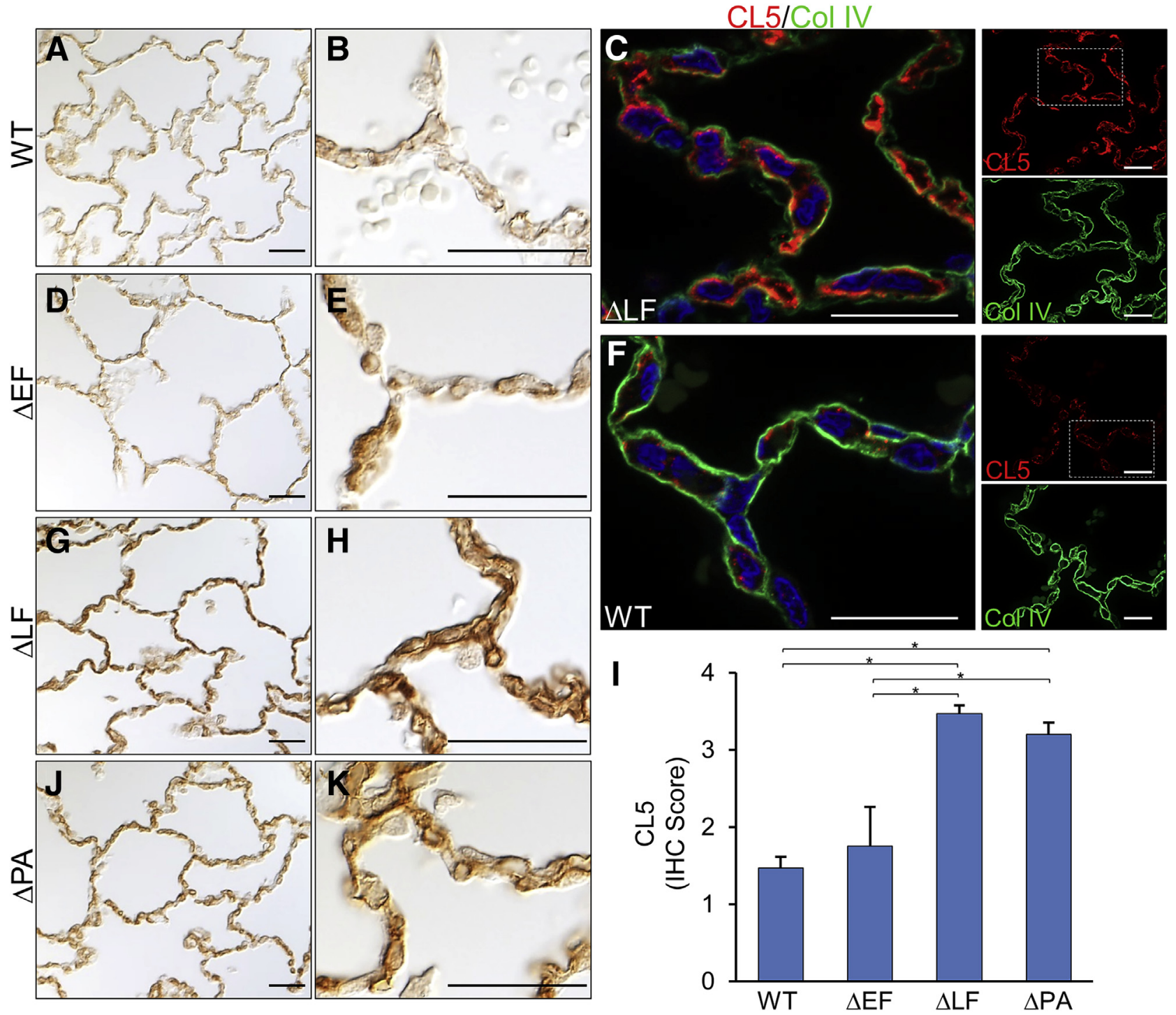

Figure 3 Claudin-5 (CL5) expression in the alveolar-capillary endothelium. Representative immunohistochemical images of endothelial CL5 expression following spore challenge with wild-type (WT) (A and B), deletion mutant strains lacking edema factor $(\Delta E F)(\mathbf{D}$ and $\mathbf{E})$, lethal factor $(\Delta \mathrm{LF})(\mathbf{G}$ and $\mathbf{H})$, or protective antigen ( $\triangle \mathrm{PA})(\mathbf{J}$ and $\mathbf{K})$. Reduced CL5 immunoreactivity (diaminobenzidine staining, brown) is observed with WT and $\triangle \mathrm{EF}$ compared with more prominent and continuous staining with $\triangle \mathrm{LF}$ and $\triangle \mathrm{PA}$. Representative dual immunofluorescence staining of CL5 (red) and collagen type IV (Col IV, green) in alveolar septa from $\Delta \mathrm{LF}(\mathbf{C})$ and WT (F) challenges. Boxed areas in the CL5 alone panels are digitally enlarged to better visualize CL5 and Col IV labeling. Nuclei were counterstained with Hoechst 33342 (blue). Semi-quantitative analysis of CL5 immunoreactivity is shown (I). Immunohistochemistry (IHC) scores are reported as the means \pm SEM for three animals per challenge group. ${ }^{*} P<0.05$. Scale bars: $20 \mu \mathrm{m}(\mathbf{A}-\mathbf{H}, \mathbf{J}$, and $\mathbf{K})$. Original magnification: $\times 400(\mathbf{A}, \mathbf{D}, \mathbf{G}$, and J); $\times 600(B, C, E, F, H$, and $\mathbf{K})$.

were co-stained with Col IV, the major collagen constituent of the alveolar basement membrane. Representative immunofluorescence staining of $\Delta \mathrm{LF}$ lungs showed uniformly expressed claudin-5 along the inner surface of the alveolar capillary loops bordered externally by the Col IV-expressing thin and thick portions of the alveolar basement membrane (Figure 3C). In WT lungs, Col IV-stained alveolar basement membrane encircled the discontinuous and punctate claudin5 expression (Figure 3F). Endothelial claudin-5 labeling was also clearly distinguishable from epithelial E-cad staining (Supplemental Figure S1).

Next, the expression of ERG, an endothelial-specific nuclear transcription factor that regulates critical homeostatic and inflammatory responses, was examined. Immunohistochemical staining identified ERG-positive alveolar capillary endothelial cells in all challenge groups (Figure 4). Semi-quantitative image analyses revealed no significant differences in the total number of ERG-positive endothelial cells between the various challenge groups (Figure 4C). Despite having similar numbers of endothelial cells, ERG staining intensity in $\triangle \mathrm{LF}$ and $\triangle \mathrm{PA}$ lungs was higher than in $\mathrm{WT}$ and $\Delta \mathrm{EF}$ lungs. High-magnification image analyses revealed a greater percentage of highintensity ERG nuclei in $\triangle \mathrm{LF}$ and $\triangle \mathrm{PA}$ lungs relative to WT and $\Delta \mathrm{EF}$ lungs, whereas WT and $\Delta \mathrm{EF}$ lungs showed a greater percentage of low-intensity ERG nuclei (Figure $4 \mathrm{H}$ ). 

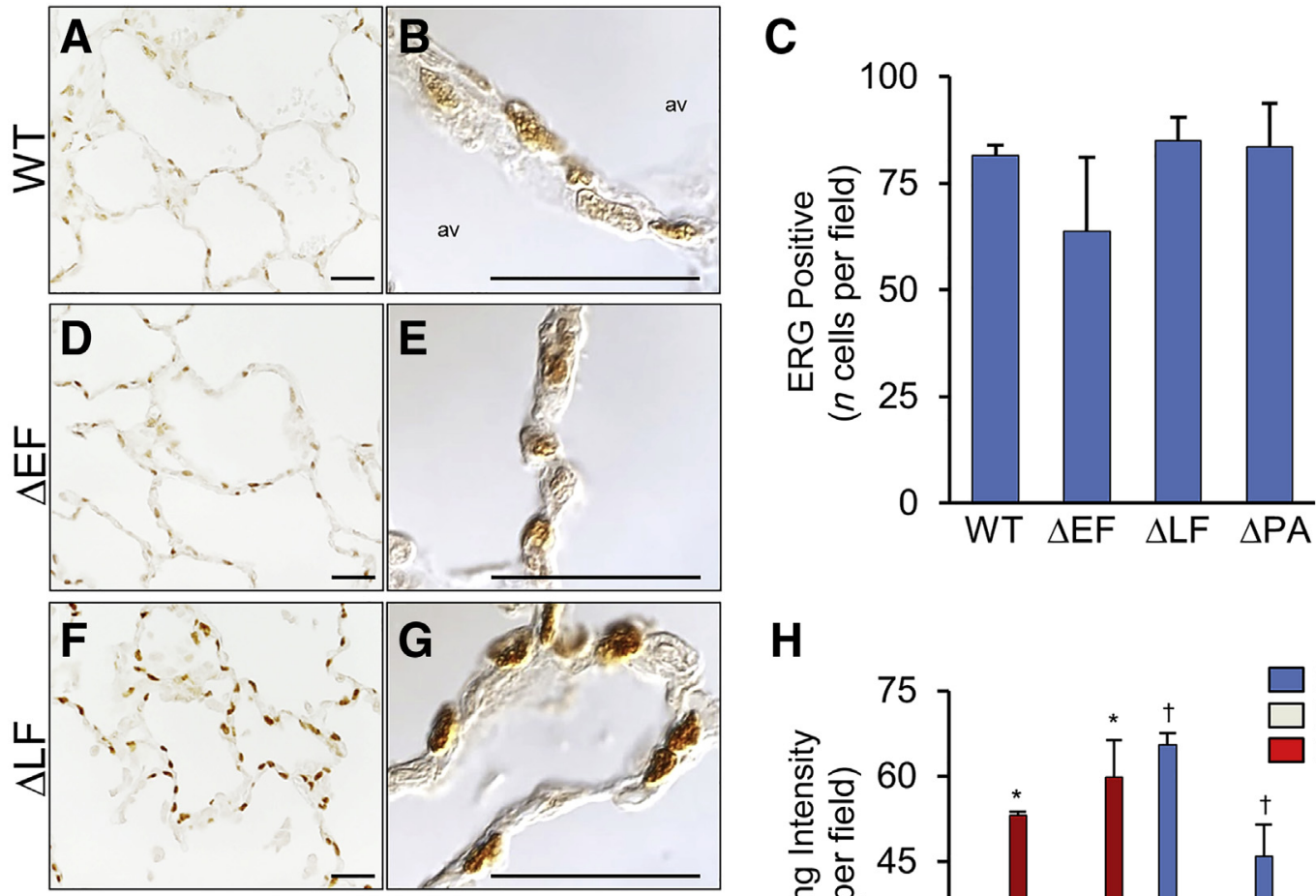

\section{H}
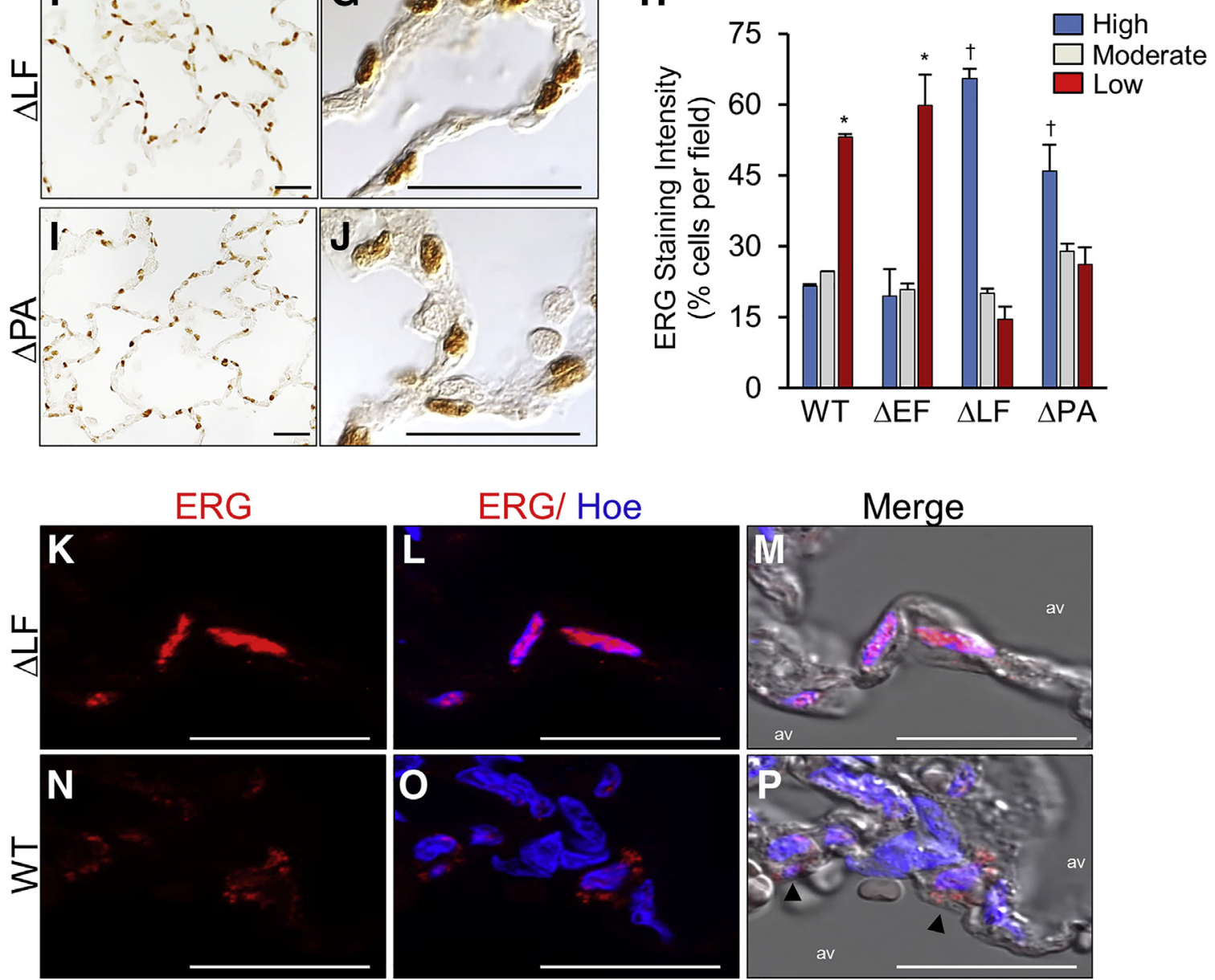

Figure 4 Endothelial ETS-related gene (ERG) expression in alveolar capillaries. Representative immunohistochemical images of endothelial ERG expression following spore challenge with Bacillus anthracis Ames strain [wild-type (WT)] strain (A and B), deletion mutant strains lacking edema factor $(\Delta \mathrm{EF})(\mathbf{D}$ and $\mathbf{E})$, lethal factor $(\Delta \mathrm{LF})(\mathbf{F}$ and $\mathbf{G})$, or protective antigen $(\Delta \mathrm{PA})$ (I and $\mathbf{J})$. Prominent endothelial-specific nuclear ERG expression (diaminobenzidine staining, brown) is observed in $\triangle \mathrm{LF}$ and $\triangle \mathrm{PA}$ compared with lower intensity expression in WT and $\Delta E F$. Semi-quantitative analyses of total ERG-stained cells and staining intensity are shown ( $\mathbf{C}$ and $\mathbf{H}$ ). Total ERG-positive cells are reported as the means \pm SEM for three animals per challenge group. ERG staining intensity was analyzed as the percentage of cells per field expressing high, moderate, or low ERG immunoreactivity. The values for each staining level are reported as the means \pm SEM for three animals per challenge group. Representative immunofluorescence images with differential interference contrast (DIC) of ERG-stained nuclei (red) show prominent nuclear localization in $\Delta$ LF (K-M) compared with reduced nuclear staining in WT (N-P, arrowheads). Nuclei were counterstained with Hoechst 33342 (Hoe, blue). ${ }^{*}<<0.05$ versus low ERG intensity $\Delta$ LF or $\Delta \mathrm{PA} ;{ }^{\dagger} P<0.05$ versus high ERG intensity WT or $\Delta$ EF. Scale bars: $20 \mu \mathrm{m}(\mathbf{A}, \mathbf{B}, \mathbf{D}-\mathbf{G}$, and $\mathbf{I}-\mathbf{P})$. Original magnification: $\times 400(\mathbf{A}, \mathbf{D}, \mathbf{F}$, and $\mathbf{I}) ; \times 1000$ $(\mathbf{B}, \mathbf{E}, \mathbf{G}$, and $\mathbf{J}) ; \times 630(\mathbf{K}-\mathbf{P})$. av, alveolar space. 
Immunofluorescence staining revealed a specific and highly concentrated nuclear localization of endothelial ERG in $\Delta$ LF lungs (Figure $4, \mathrm{~K}-\mathrm{M}$ ) compared with a weaker and punctate extranuclear ERG signal in WT endothelial cells (Figure 4, N-P). This observation suggests that the reduced ERG staining intensity may be indicative of decreased ERG expression levels and/or altered subcellular localization.

\section{Alveolar Septal Accumulation of Neutrophils after WT and $\Delta E F$ Challenge}

Because dysregulated inflammatory responses may contribute to alveolar-capillary barrier dysfunction, neutrophil recruitment was examined by analyzing the expression of NE and MPO, two major enzymes expressed in the azurophilic granules of neutrophils (Figure 5, A-L). Semi-quantitative analyses of immunohistochemical staining revealed extensive accumulation of NE- and MPOpositive neutrophils in WT and $\Delta \mathrm{EF}$ lungs relative to $\triangle \mathrm{LF}$ and $\triangle \mathrm{PA}$ lungs (Figure 5, M and $\mathrm{N}$ ). The neutrophils were predominantly trapped in the alveolar septa with minimal entry into the intra-alveolar compartment. Although WT and $\triangle \mathrm{EF}$ exhibited a similar total number of NE- and MPOpositive cells, WT neutrophils expressed a more diffuse and heterogeneous NE and MPO staining pattern, possibly related to differences in cell morphology (Figure 5, A-C). In addition to the NE- and MPO-positive granule-type staining in neutrophils, greater extracellular MPO staining of alveolar septa in WT lungs compared with the other three groups was often detected (Figure $5 \mathrm{C}$ ).

To further investigate neutrophil recruitment processes, dual-immunolabeling for MPO and the complement activation product $\mathrm{C} 5 \mathrm{a}$, a potent chemoattractant and functional modulator of neutrophils, was performed. In WT lungs, C5a was detected in MPO-positive neutrophils and, notably, appeared as intense punctate and diffuse immunoreactivity within the alveolar septa (Figure 6, A-D). The septal C5a punctate deposits were often located nearby bacterial aggregates (Figure 6, Q and R). In contrast to WT, $\Delta \mathrm{EF}$ lungs showed MPO-positive neutrophils typically co-expressing a more homogeneously distributed C5a signal as well as less punctate or diffuse C5a alveolar septal staining (Figure 6, $\mathrm{E}-\mathrm{H})$. In $\Delta \mathrm{LF}$ and $\Delta \mathrm{PA}$ lungs, C5a was mainly observed in the scattered MPO-positive cells with less punctate staining than WT (Figure 6, I-P). These findings suggest that complement-related processes play a role in the inflammatory responses at the level of the alveolar-capillary barrier.

\section{Alveolar Expression of Coagulation and Complement Markers}

To investigate the potential relationship between complement activation and aberrant coagulation processes, the expression of complement $\mathrm{C} 9, \mathrm{TF}$, and fibrin were analyzed by immunohistochemistry and immunofluorescence. In serially stained sections, WT lungs showed multifocal fibrin deposits along the apical lining of alveolar epithelial surfaces and intra-alveolar spaces, which consistently co-localized with high levels of TF and $\mathrm{C} 9$ (Figure 7, A-C). $\Delta \mathrm{EF}$ lungs exhibited fewer intra-alveolar fibrin deposits and lower intraalveolar expression of TF and C9 (Figure 7, D-F). In $\Delta \mathrm{LF}$ and $\triangle \mathrm{PA}$ lungs, intra-alveolar expression of fibrin, $\mathrm{TF}$, or $\mathrm{C} 9$ was absent (Figure 7, G-L). In WT lungs, highmagnification immunofluorescence analysis further highlighted the strong co-localization of TF and C9 in the fibrinous intra-alveolar material in focally damaged alveoli (Figure 8, A-C). Dual labeling for TF and C5b9 produced similar results (Supplemental Figure S2). In contrast, the intra-alveolar material occasionally observed in $\Delta \mathrm{EF}$ lungs typically contained histiocyte-like aggregates and expressed lower levels of TF and C9 (Figure 8, D-F). In WT lungs, TF expression was primarily detected in intra-alveolar spaces and was not markedly expressed in the alveolar capillaries or interstitium. In contrast to TF, WT lungs showed notable diffuse C9 immunoreactivity in the alveolar septa, whereas minimal septal $\mathrm{C} 9$ expression was detected in $\triangle \mathrm{EF}, \Delta \mathrm{LF}$, and $\triangle$ PA (Figure $8, \mathrm{G}-\mathrm{J}$ ). These observations suggest that a close interplay between the complement and coagulation pathways may contribute to the overall host response to infection within the alveolar-capillary compartment.

\section{Discussion}

Respiratory distress is a common cause of mortality in anthrax patients, which emphasizes the need to better understand the role of bacterial virulence factors as well as the pathogenic events leading to pulmonary dysfunction. Anthrax toxemia is widely believed to play a critical yet incompletely defined role during the early and late stages of the disease. ${ }^{1-3}$ Emerging evidence suggests that anthrax sepsis possibly facilitated by toxin-mediated innate immune dysfunction triggers the full range of pathophysiologic features associated with anthrax lethality. ${ }^{1,30,33}$ This scenario highlights the value of studying the actions of toxins in fully virulent bacterial spore challenge models that take into consideration toxin production in vivo, the presence of other bacterial virulence factors or pathogen-associated molecular patterns, and the impact of host responses to an actual infection. ${ }^{18,29,30,36,37}$ The current study identified structural and inflammatory changes at the level of the alveolar-capillary barrier in cynomolgus monkeys challenged with the fully virulent $B$. anthracis Ames WT strain (see Figure 9 for a schematic summary). These findings also support a critical role for LT in the development of systemic disease and pulmonary pathophysiology, whereas ET may aggravate the pathologic severity of an established infection.

Massive bacteremia with hematogenous spreading to organs characterizes the late stages of inhalational anthrax. ${ }^{15-18}$ Significant deposition of vegetative bacilli was observed in the terminal lungs of monkeys challenged with $\mathrm{WT}$ and $\triangle \mathrm{EF}$ but not with $\Delta \mathrm{LF}$ or $\Delta \mathrm{PA}$. These 

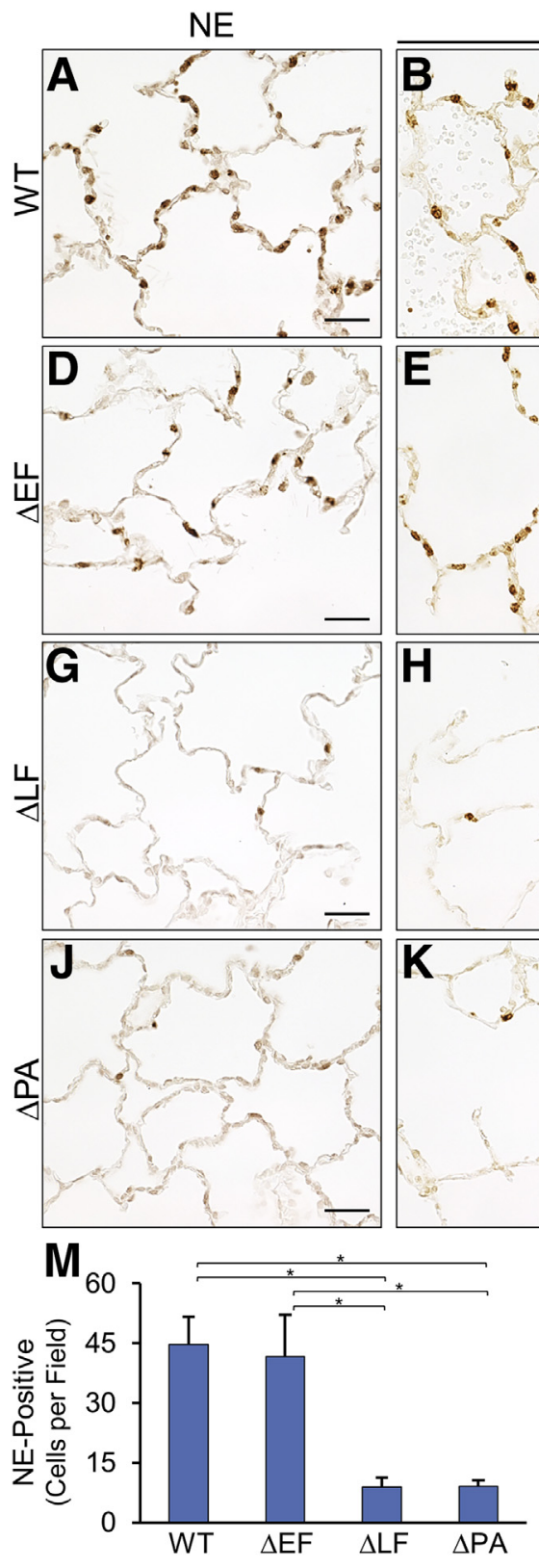

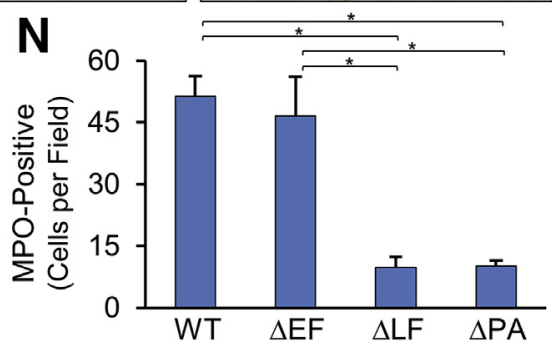

\section{MPO}
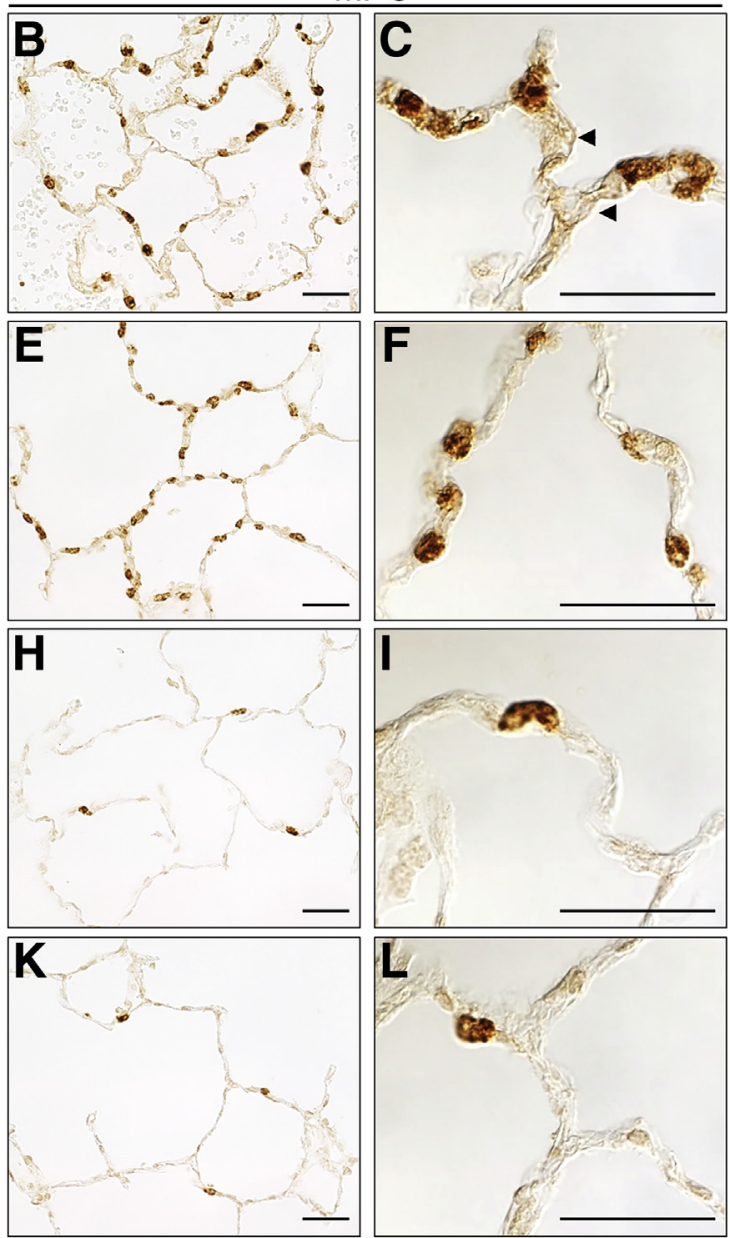

Figure 5 Neutrophil sequestration in alveolar septa. A-L: Representative immunohistochemical images of neutrophil elastase (NE) and myeloperoxidase (MPO) following spore challenge with Bacillus anthracis Ames strain [wild-type (WT)] strain $(\mathbf{A}-\mathbf{C})$, deletion mutant strains lacking edema factor $(\Delta \mathrm{EF})(\mathbf{D}-\mathbf{F})$, lethal factor $(\Delta \mathrm{LF})$ $(\mathbf{G}-\mathbf{I})$, or protective antigen $(\triangle \mathrm{PA})(\mathbf{J}-\mathbf{L})$. Arrowheads in (C) denote detectable MPO staining of alveolar walls. $\mathrm{M}$ and $\mathrm{N}$ : Semi-quantitative analyses of NE and MPO-positive cells. Values are reported as the means \pm SEM for three animals per challenge group. ${ }^{*} P<0.05$. Scale bars: $20 \mu \mathrm{m}$ (A-L). Original magnification: $\times 400($ A, B, D, E, $\mathbf{G}, \mathbf{H}, \mathbf{J}$, and $\mathbf{K}) ; \times 1000(\mathbf{C}, \mathbf{F}, \mathbf{I}$, and $\mathbf{L})$. observations are consistent with previously reported findings that LT plays a determinative role in bacterial growth, survival, and dissemination in this spore challenge model. ${ }^{34}$ Notably, WT lungs exhibited more extensive bacterial deposition than $\triangle \mathrm{EF}$, suggesting that the presence of ET may facilitate bacterial propagation and/or survival. Together, these findings are consistent with the reported ability of LT and ET to impair host defenses against the bacteria. ${ }^{1,2,11-14}$ Bacterial aggregates accumulated predominantly in the alveolar septa, as reported in clinical cases and other experimental models of anthrax. ${ }^{15,16,18,38}$ Both WT and $\triangle \mathrm{EF}$ challenges were $100 \%$ lethal, whereas $\Delta \mathrm{LF}$ and $\triangle \mathrm{PA}$ were nonlethal and produced minimal histopathology. It should be noted, however, that this retrospective histologic comparison was limited to lung specimens that were not time-matched given that surviving animals were euthanized at 14 days $(\triangle \mathrm{PA})$ and 21 days $(\Delta \mathrm{LF})$ after the challenge, whereas WT and $\Delta \mathrm{EF}$ animals were moribund or dead within 2 to 5 days. WT lungs generally showed more extensive histopathologic changes than $\Delta \mathrm{EF}$ lungs, particularly in relation to intra-alveolar hemorrhages. Further investigation is needed to clarify the extent to which the contributory role of ET in this setting reflects its direct pathogenic effects and/or dysregulated host responses to the increased bacterial burden.

Proper lung function depends on the structural integrity of the alveolar-capillary barrier, which is maintained by intercellular adherens junctions and tight junctions that 

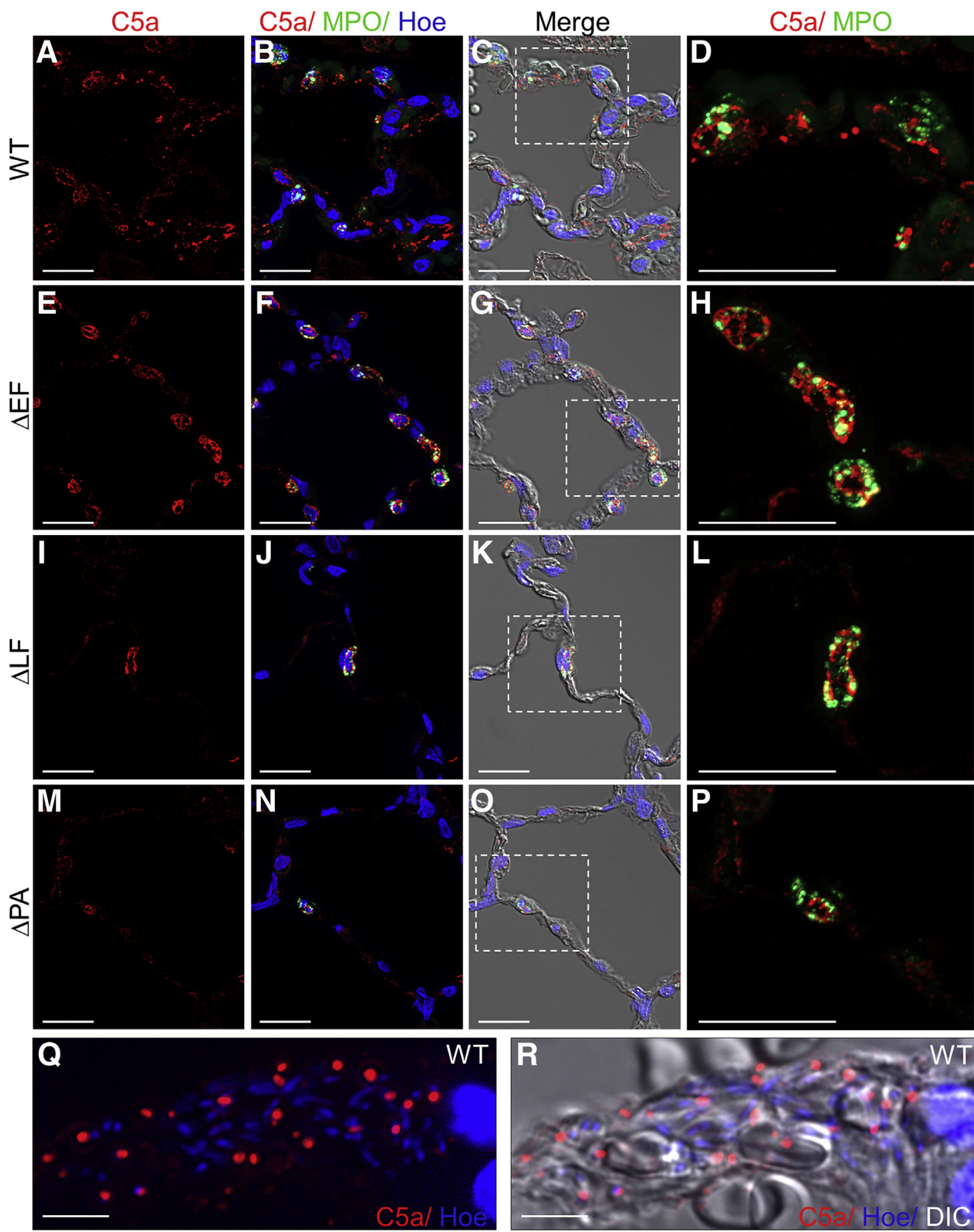

Figure 6 Alveolar localization of myeloperoxidase (MP0) and complement C5a expression. A-P: Representative immunofluorescence images of MP0 (green) and complement C5a (red) merged with differential interference contrast (DIC) in monkeys challenged with Bacillus anthracis Ames strain [wild-type (WT)] strain (A-D), deletion mutant strains lacking edema factor $(\Delta \mathrm{EF})(\mathbf{E}-\mathbf{H})$, lethal factor $(\Delta \mathrm{LF})(\mathbf{I}-\mathbf{L})$, or protective antigen $(\triangle \mathrm{PA})(\mathbf{M}-\mathbf{P})$. Nuclei were counterstained with Hoechst 33342 (Hoe, blue). Images are constructed from Z-stack slices collected at $0.6-\mu \mathrm{m}$ intervals $(6 \mu \mathrm{m}$ thickness in total) and visualized as maximum intensity projections in orthogonal mode. Boxed areas in each of the DIC merge images were digitally enlarged to better visualize MPO and C5a labeling of alveolar septa (D, H, L, and P). Q and R: Immunofluorescence combined with DIC imaging of C5a punctate (red) in the vicinity of bacterial aggregates (blue) in WT alveolar septa. The exposure setting for the Hoe dye is increased to allow the detection of the bacilli rods trapped in the septa. Scale bars: $20 \mu \mathrm{m}(\mathbf{A}-\mathbf{P}) ; 5 \mu \mathrm{m}(\mathbf{Q}$ and $\mathbf{R})$. Original magnification: $\times 630(\mathbf{A}-\mathbf{R})$.

regulate paracellular permeability, alveolar fluid balance, and optimal gas exchange. ${ }^{19-22}$ Previous cell culture studies have shown that purified LT can reduce E-cad and claudin-5 expression and decrease epithelial and endothelial barrier function. $^{24-29}$ Here, in a pathogenically relevant spore challenge model, diminished E-cad and claudin-5 expression were observed in WT and $\Delta \mathrm{EF}$ lungs indicative of compromised alveolar epithelial and endothelial junctional 

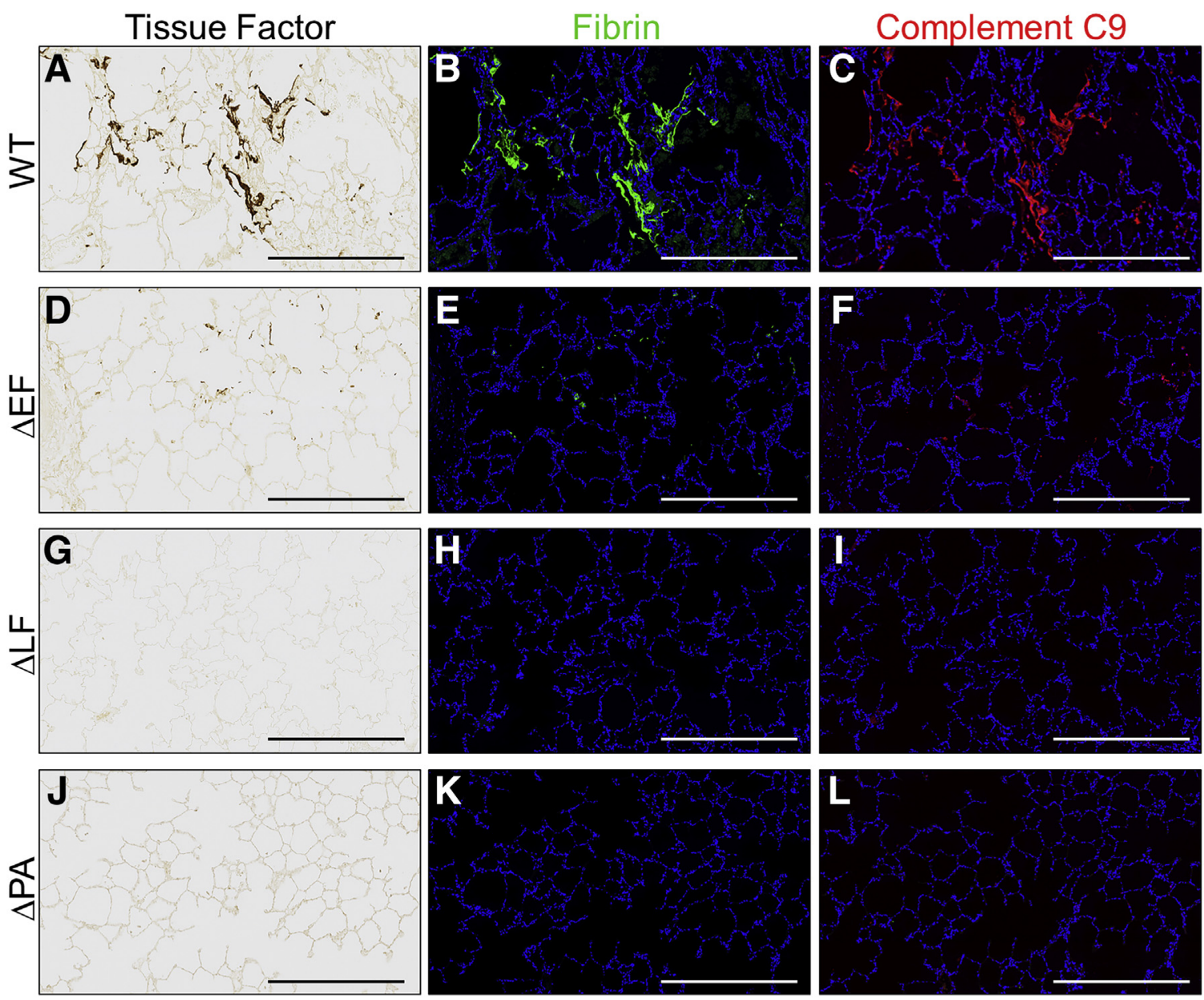

Figure 7 Alveolar co-localization of tissue factor (TF), complement component 9 (C9), and fibrin. Serial monkey lung following spore challenge with Bacillus anthracis Ames strain [wild-type (WT)] strain $(\mathbf{A}-\mathbf{C})$, deletion mutant strains lacking edema factor $(\Delta \mathrm{EF})(\mathbf{D}-\mathbf{F})$, lethal factor $(\Delta \mathrm{LF})(\mathbf{G}-\mathbf{I})$, or protective antigen $(\triangle P A)(\mathbf{J}-\mathbf{L})$ were stained for TF, complement C9, and fibrin by immunohistochemistry (TF; brown) and immunofluorescence (C9, red; fibrin, green). Nuclei were counterstained with Hoechst 33342 (blue). Scale bars: $500 \mu \mathrm{m}$. Original magnification: $\times 20$.

integrity. Interestingly, WT and $\Delta \mathrm{EF}$ lungs exhibited similar E-cad and claudin-5 staining, suggesting that the observed enhancement of WT lung histopathology by ET involves processes that occur independently or secondary to the disruption of junctional complexes.

ERG, a major ETS-family transcription factor highly enriched in endothelial cells, plays a critical role in the regulation of vascular permeability, junctional stability, inflammation, and angiogenesis. ${ }^{39}$ ERG upregulates the synthesis of specific endothelial proteins, including claudin5 and VE-cadherin, while repressing the expression of the proinflammatory proteins IL-8 and intercellular adhesion molecule $1 .^{39-41}$ The current study found no significant differences in the total number of ERG-positive alveolar endothelial cells between the various challenge groups. WT and $\triangle \mathrm{EF}$ alveolar endothelial cells, however, displayed a marked reduction in nuclear ERG immunoreactivity compared with $\triangle \mathrm{LF}$ and $\triangle \mathrm{PA}$. This outcome implies that the reduced claudin- 5 expression in $\mathrm{WT}$ and $\Delta \mathrm{EF}$ lungs may be related to altered claudin-5 synthesis or degradation rather than to a loss of alveolar endothelial cells. Given that ERG is a key transcriptional regulator of the CLDN5 gene, we speculate that the diminished ERG expression or altered subcellular localization may contribute to reduced claudin-5 synthesis. ${ }^{39,41}$ Previous studies have also shown that proinflammatory stimuli, as well as experimental murine sepsis, markedly suppress endothelial ERG expression, raising the possibility that anthrax-related inflammation plays a role in the reduced ERG expression in WT and $\Delta \mathrm{EF}$ alveolar endothelial cells. ${ }^{39,40}$

The loss of alveolar junctional integrity may be the consequence of direct interactions with $B$. anthracis virulence factors and/or dysregulated host responses to infection. Neutrophils are critical primary responders of the innate immune system that attack bacteria using reactive oxygen species, proteases, antimicrobial peptides, and neutrophil extracellular traps. ${ }^{42-44}$ Despite this major role, excessive alveolar neutrophil recruitment and activation often contribute to the alveolar-capillary barrier dysfunction in acute lung injury, acute respiratory distress 

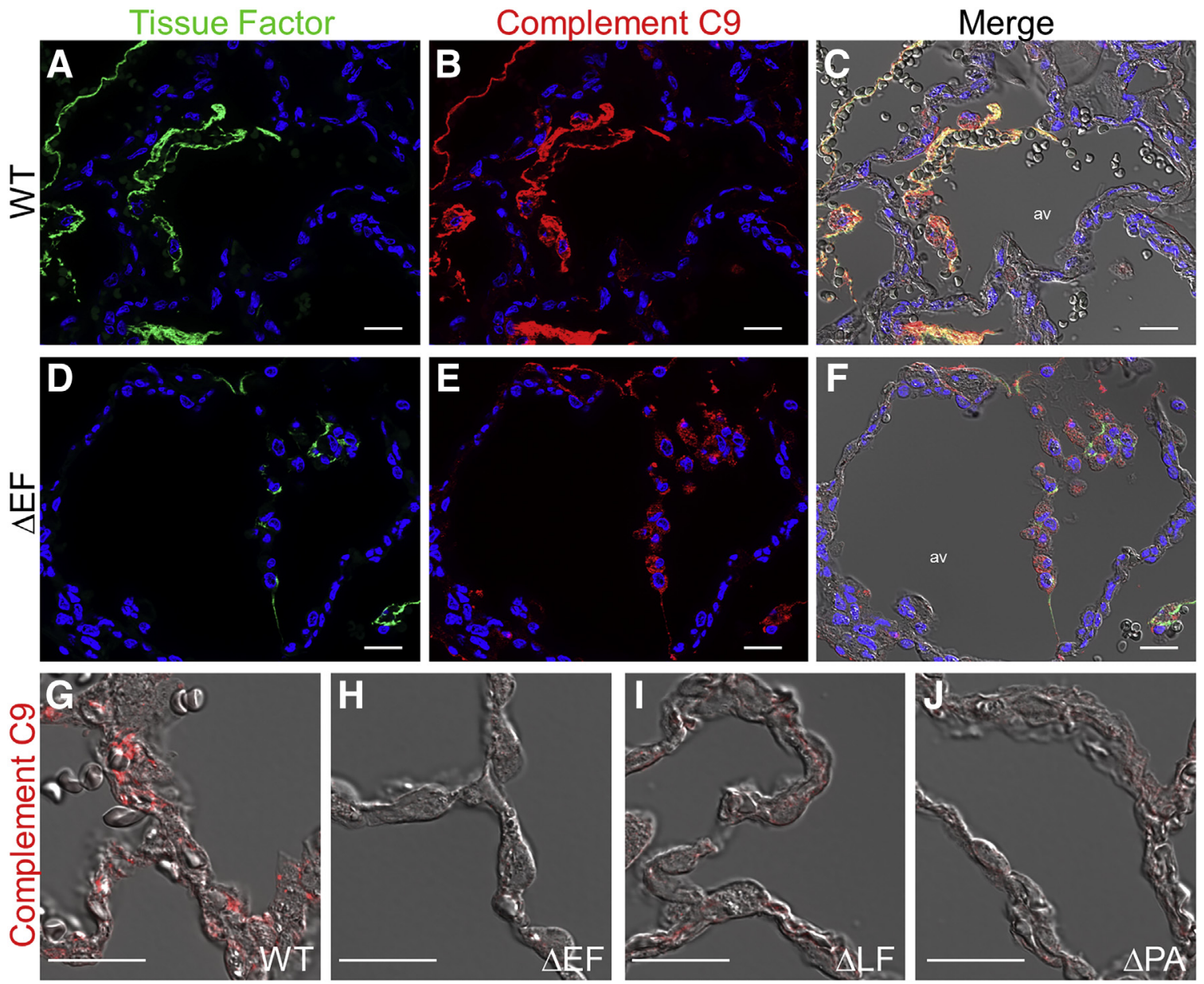

Figure 8 Alveolar expression of tissue factor (TF) and complement component 9 (C9). Representative immunofluorescence images of TF (green) and C9 (red) merged with differential interference contrast in monkey lungs following spore challenge with Bacillus anthracis Ames strain [wild-type (WT)] (A-C) and deletion mutant stain lacking edema factor $(\Delta \mathrm{EF})(\mathbf{D}-\mathbf{F})$. Alveolar septa staining for $C 9$ (red) shows prominent labeling in WT (G) compared with minimal signal in $\Delta \mathrm{EF}$, lethal factor $(\Delta \mathrm{LF})$, and protective antigen $(\Delta \mathrm{PA})(\mathbf{H}-\mathbf{J})$. Nuclei were counterstained with Hoechst 33342 (blue). Scale bars: $20 \mu \mathrm{m}$. 0 riginal magnification: $\times 630$. av, alveolar space.

syndrome, and microbial infections. ${ }^{42-44}$ With respect to anthrax infection, an effective neutrophil response is believed to be critical to host survival. ${ }^{45,46}$ The current study found marked alveolar septal accumulation of NE- and MPO-positive neutrophils in WT and $\Delta \mathrm{EF}$ lungs, with minimal distribution in alveolar spaces consistent with previous clinical and experimental observations. ${ }^{15,16,18}$ Similar numbers of neutrophils were present in WT and $\Delta \mathrm{EF}$ lungs, although neutrophil quantitation in WT lungs was complicated by the irregular and diffusely distributed NE and MPO staining appearance. The visible differences in $\mathrm{NE}$ and MPO labeling between WT and $\triangle \mathrm{EF}$ lungs may be indicative of differences in neutrophil activation, degranulation, neutrophil extracellular trap release, and/or death. ${ }^{42}$ The greater bacterial burden in WT lungs, relative to $\Delta \mathrm{EF}$, suggests that ET may facilitate bacilli growth and/or survival in WT lungs, possibly by reducing the efficiency of neutrophil bactericidal activity. ${ }^{12,14}$ Dysregulated neutrophil functions mediated by the coordinated actions of LT and ET could contribute directly or indirectly to lung-related pathogenesis. $^{1,2,12-14,45}$
The complement system is a central effector of innate immunity that functions in the recognition, marking, and elimination of pathogens. ${ }^{47}$ Complement activation via the classical, lectin, or alternative pathways results in the formation of the anaphylatoxins C3a and C5a as well as the lytic membrane attack complex (C5b9). C5a is also generated directly or indirectly by activated neutrophils, macrophages, platelets, and procoagulant proteins. $^{48,49}$ The primary C5a receptor (C5aR) is expressed on the surface of neutrophils and other inflammatory and noninflammatory cells, including both endothelial and epithelial cells. ${ }^{49,50}$ C5a serves as a particularly potent chemoattractant for neutrophils and other inflammatory cells, activates neutrophil release of granule-based enzymes, stimulates reactive oxygen species and cytokine production, promotes the formation of neutrophil extracellular traps, and increases vascular permeability. ${ }^{48-50}$ Basal C5a levels may accelerate neutrophil priming as part of an effective innate immune response to infections. Excess C5a production, however, induces neutrophil dysfunction characterized by abnormal chemotaxis, reduced reactive oxygen species production, 


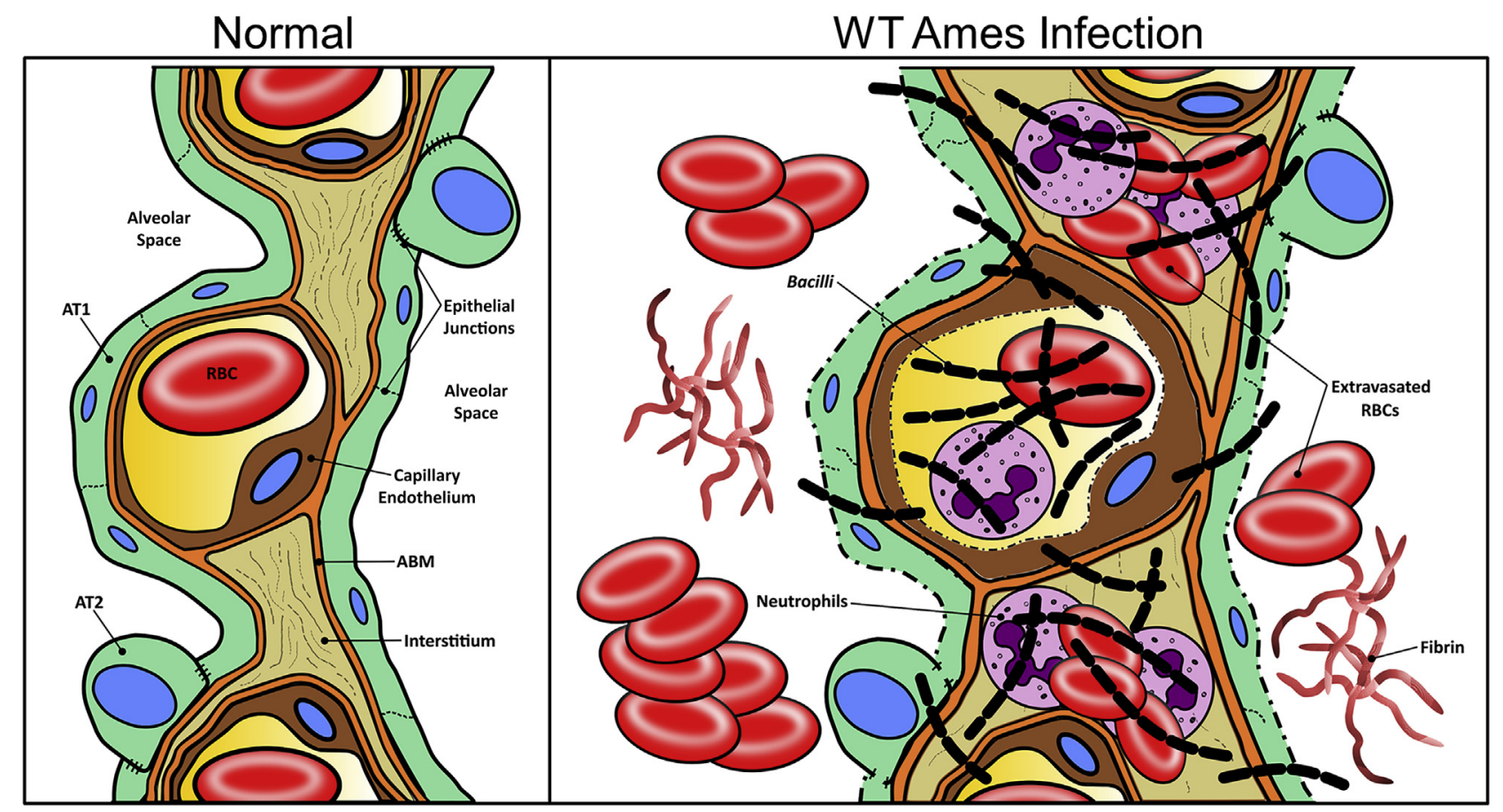

Figure 9 Schematic representation of the alveolar-capillary barrier. Left panel: The normal alveolar-capillary barrier is formed by: i) alveolar epithelium consisting of alveolar epithelial type 1 (AT1) and type 2 (AT2) cells closely interacting with each other through intercellular adherens junctions (AJs) and tight junctions (TJs) that control barrier permeability, ii) alveolar capillary endothelium also stabilized by barrier-regulating AJs and TJs, and iii) the alveolar basement membrane (ABM) that separates both epithelial and endothelial layers and supports alveolar structural stability. Right panel: Pathologic changes to the alveolar-capillary barrier after infection with the fully virulent Bacillus anthracis Ames wild-type (WT) strain include: i) widened and/or damaged alveolar septa, ii) extensive capillary congestion and interstitial extravasation of red blood cells (RBCs), iii) alveolar septal sequestration of neutrophils and anthrax bacilli, iv) diffuse destabilization of epithelial and endothelial intercellular junctions, v) dysregulated complement and coagulation processes, and vi) intraalveolar hemorrhages and fibrin deposition.

and defective phagocytosis. In general, overactivation of the complement system magnifies the excessive inflammatory response associated with sepsis. In the current study, both WT and $\triangle \mathrm{EF}$ lungs showed increased alveolar C5a expression relative to $\triangle \mathrm{LF}$ and $\triangle \mathrm{PA}$ lungs, suggesting a potential role for $\mathrm{C} 5 \mathrm{a}$ in the host response to anthrax infection. Notably, in WT lungs, C5a was expressed in MPO-positive neutrophils and in a diffuse punctate pattern throughout the alveolar septa, whereas it was predominately localized to MPO-positive neutrophils in $\triangle \mathrm{EF}$ lungs. The significance of this differential C5a expression pattern is not yet clear, although it raises the possibility that ET may directly or indirectly modulate C5a-related cellular interactions or signaling in the alveolar compartment. The finding that complement punctate deposits often localized with bacilli clusters in the alveolar septa may reflect possible complement deposition on neutrophil extracellular traps, as previously described. ${ }^{51}$

Significant cross-talk between the complement and coagulation systems profoundly affects the innate immune response to microbial infections. ${ }^{52,53}$ For example, C5a has been implicated in the TF-mediated procoagulant response associated with severe sepsis, and blocking C5a signaling improves the treatment of microbial-induced acute lung injury. ${ }^{54,55}$ Clinical and experimental anthrax infection has been linked to disseminated intravascular coagulation, thrombosis, and fibrin deposition. ${ }^{15-18,30,33}$ The current study identified multifocal areas of intraalveolar fibrin deposition in WT lungs co-localized with $\mathrm{TF}, \mathrm{C} 9$, and $\mathrm{C} 5 \mathrm{~b} 9$ consistent with the reported interplay between these pathways. ${ }^{52,53}$ In contrast, these markers were far less evident in $\triangle \mathrm{EF}$ lungs and absent in $\Delta \mathrm{LF}$ and $\Delta \mathrm{PA}$ lungs. WT lungs also exhibited greater alveolar C9 septal deposition than $\triangle \mathrm{EF}$ lungs. Upregulated $\mathrm{TF}$ is typically considered a consequence of endothelial injury or derived from activated neutrophils or monocytes. ${ }^{52,53,56}$ In this study, however, TF expression in WT lungs was primarily detected in intra-alveolar fibrin deposits or along the inner alveolar epithelial lining with minimal TF labeling of endothelium or neutrophils. Previous studies have proposed that epithelial-derived TF plays a predominant role in procoagulant processes in the lung ${ }^{57}$; nevertheless, the current findings do not rule out the potential contribution from other sources. Further disease progression studies are needed to more completely evaluate the complex temporal interplay between inflammation, complement, and coagulation activation and their potential pathologic implications.

In conclusion, our retrospective investigation of archival lung specimens from a pathologically relevant 
nonhuman primate model challenged with fully virulent B. anthracis spores identified a series of structural changes to the alveolar-capillary barrier and their potential association with activated innate immune and procoagulant pathways. Challenges with isogenic toxindeficient mutant strains further revealed that functional $\mathrm{LT}$ is required to trigger systemic disease, pulmonary pathologies, and death, whereas ET may aggravate some of the observed pulmonary pathologies but is not ultimately required for lethality. Together, these findings provide new insight on the potential events underlying the profound pulmonary pathologies of systemic anthrax disease and may help better define the relative pathogenic contributions of LT and ET in the context of a fully virulent $B$. anthracis infection.

\section{Acknowledgment}

We thank Julie A. Hutt (formerly at LRRI) for providing the lung specimens.

\section{Supplemental Data}

Supplemental material for this article can be found at http://doi.org/10.1016/j.ajpath.2020.06.007.

\section{References}

1. Moayeri M, Leppla SH, Vrentas C, Pomerantsev AP, Liu S: Anthrax pathogenesis. Annu Rev Microbiol 2015, 69:185-208

2. Goossens PL, Tournier JN: Crossing of the epithelial barriers by Bacillus anthracis: the known and the unknown. Front Microbiol 2015, 6:1122

3. Friebe S, van der Goot FG, Bürgi J: The ins and outs of anthrax toxin. Toxins (Basel) 2016, 8:69

4. Leppla S: Anthrax toxin edema factor: a bacterial adenylate cyclase that increases cyclic AMP concentrations of eukaryotic cells. Proc Natl Acad Sci U S A 1982, 79:3162-3166

5. Pezard C, Berche P, Mock M: Contribution of individual toxin components to virulence of Bacillus anthracis. Infect Immun 1991, 59:3472-3477

6. Moayeri M, Haines D, Young HA, Leppla SH: Bacillus anthracis lethal toxin induces TNF-alpha-independent hypoxia-mediated toxicity in mice. J Clin Invest 2003, 112:670-682

7. Firoved AM, Miller GF, Moayeri M, Kakkar R, Shen Y, Wiggins JF, McNally EM, Tang WJ, Leppla SH: Bacillus anthracis edema toxin causes extensive tissue lesions and rapid lethality in mice. Am J Pathol 2005, 167:1309-1320

8. Suffredini DA, Li Y, Xu W, Moayeri M, Leppla S, Fitz Y, Cui X, Eichacker PQ: Shock and lethality with anthrax edema toxin in rats are associated with reduced arterial responsiveness to phenylephrine and are reversed with adefovir. Am J Physiol Heart Circ Physiol 2017, 313:H946-H958

9. Liu S, Zhang Y, Moayeri M, Liu J, Crown D, Fattah RJ, Wein AN, Yu ZX, Finkel T, Leppla SH: Key tissue targets responsible for anthrax-toxin-induced lethality. Nature 2013, 501:63-68

10. Ebrahimi CM, Sheen TR, Renken CW, Gottlieb RA, Doran KS: Contribution of lethal toxin and edema toxin to the pathogenesis of anthrax meningitis. Infect Immun 2011, 79:2510-2518
11. Cleret-Buhot A, Mathieu J, Tournier JN, Quesnel-Hellmann A: Both lethal and edema toxins of Bacillus anthracis disrupt the human dendritic cell chemokine network. PLoS One 2012, 7:e43266

12. Szarowicz SE, During RL, Li W, Quinn CP, Tang WJ, Southwick FS: Bacillus anthracis edema toxin impairs neutrophil actin-based motility. Infect Immun 2009, 77:2455-2464

13. Weiner ZP, Ernst SM, Boyer AE, Gallegos-Candela M, Barr JR, Glomski IJ: Circulating lethal toxin decreases the ability of neutrophils to respond to Bacillus anthracis. Cell Microbiol 2014, 16: 504-518

14. Crawford MA, Aylott CV, Bourdeau RW, Bokoch GM: Bacillus anthracis toxins inhibit human neutrophil NADPH oxidase activity. J Immunol 2006, 176:7557-7565

15. Guarner J, Jernigan JA, Shieh WJ, Tatti K, Flannagan LM, Stephens DS, Popovic T, Ashford DA, Perkins BA, Zaki SR; Inhalational Anthrax Pathology Working Group: Pathology and pathogenesis of bioterrorism-related inhalational anthrax. Am J Pathol 2003, 163:701-709

16. Grinberg LM, Abramova FA, Yampolskaya OV, Walker DH, Smith JH: Quantitative pathology of inhalational anthrax I: quantitative microscopic findings. Mod Pathol 2001, 14:482-495

17. Dalldorf FG, Beall FA: Capillary thrombosis as a cause of death in experimental anthrax. Arch Pathol 1967, 83:154-161

18. Welkos S, Bozue J, Twenhafel N, Cote C: Animal models for the pathogenesis, treatment, and prevention of infection by Bacillus anthracis. Microbiol Spectr 2015, 3

19. Nova Z, Skovierova H, Calkovska A: Alveolar-capillary membranerelated pulmonary cells as a target in endotoxin-induced acute lung injury. Int J Mol Sci 2019, 20:831

20. Knudsen L, Ochs M: The micromechanics of lung alveoli: structure and function of surfactant and tissue components. Histochem Cell Biol 2018, 150:661-676

21. Rezaee F, Georas SN: Breaking barriers. New insights into airway epithelial barrier function in health and disease. Am J Respir Cell Mol Biol 2014, 50:857-869

22. Kása A, Csortos C, Verin AD: Cytoskeletal mechanisms regulating vascular endothelial barrier function in response to acute lung injury. Tissue Barriers 2015, 3:e974448

23. Englert JA, Bobba C, Baron RM: Integrating molecular pathogenesis and clinical translation in sepsis-induced acute respiratory distress syndrome. JCI Insight 2019, 4:124061

24. Warfel JM, Steele AD, D'Agnillo F: Anthrax lethal toxin induces endothelial barrier dysfunction. Am J Pathol 2005, 166 1871-1881

25. Warfel JM, D’Agnillo F: Anthrax lethal toxin-mediated disruption of endothelial VE-cadherin is attenuated by inhibition of the Rho-associated kinase pathway. Toxins (Basel) 2011, 3: $1278-1293$

26. D'Agnillo F, Williams MC, Moayeri M, Warfel JM: Anthrax lethal toxin downregulates claudin-5 expression in human endothelial tight junctions. PLoS One 2013, 8:e62576

27. Langer M, Duggan ES, Booth JL, Patel VI, Zander RA, SilasiMansat R, Ramani V, Veres TZ, Prenzler F, Sewald K, Williams DM, Coggeshall KM, Awasthi S, Lupu F, Burian D, Ballard JD, Braun A, Metcalf JP: Bacillus anthracis lethal toxin reduces human alveolar epithelial barrier function. Infect Immun 2012, 80:4374-4387

28. Lehmann M, Noack D, Wood M, Perego M, Knaus UG: Lung epithelial injury by B. anthracis lethal toxin is caused by MKKdependent loss of cytoskeletal integrity. PLoS One 2009, 4:e4755

29. Popova TG, Millis B, Bradburne C, Nazarenko S, Bailey C, Chandhoke V, Popov SG: Acceleration of epithelial cell syndecan-1 shedding by anthrax hemolytic virulence factors. BMC Microbiol 2006, 6:8

30. Coggeshall KM, Lupu F, Ballard J, Metcalf JP, James JA, Farris D, Kurosawa S: The sepsis model: an emerging hypothesis for the lethality of inhalation anthrax. J Cell Mol Med 2013, 17:914-920 
31. Fritz DL, Jaax NK, Lawrence WB, Davis KJ, Pitt ML, Ezzell JW, Friedlander AM: Pathology of experimental inhalation anthrax in the rhesus monkey. Lab Invest 1995, 73:691-702

32. Vasconcelos D, Barnewall R, Babin M, Hunt R, Estep J, Nielsen C, Carnes R, Carney J: Pathology of inhalation anthrax in cynomolgus monkeys (Macaca fascicularis). Lab Invest 2003, 83:1201-1209

33. Stearns-Kurosawa D, Lupu F, Taylor FJ Jr, Kinasewitz G, Kurosawa S: Sepsis and pathophysiology of anthrax in a nonhuman primate model. Am J Pathol 2006, 169:433-444

34. Hutt JA, Lovchik JA, Drysdale M, Sherwood RL, Brasel T, Lipscomb MF, Lyons CR: Lethal factor, but not edema factor, is required to cause fatal anthrax in cynomolgus macaques after pulmonary spore challenge. Am J Pathol 2014, 184:3205-3216

35. Committee for the Update of the Guide for the Care and Use of Laboratory Animals; National Research Council: Guide for the Care and Use of Laboratory Animals. ed 8. Washington, DC, National Academies Press, 2011

36. Popescu NI, Silasi R, Keshari RS, Girton A, Burgett T, Zeerleder SS, Gailani D, Gruber A, Lupu F, Coggeshall KM: Peptidoglycan induces disseminated intravascular coagulation in baboons through activation of both coagulation pathways. Blood 2018, 132:849-860

37. Qiu P, Li Y, Shiloach J, Cui X, Sun J, Trinh L, Kubler-Kielb J, Vinogradov E, Mani H, Al-Hamad M, Fitz Y, Eichacker PQ: Bacillus anthracis cell wall peptidoglycan but not lethal or edema toxins produces changes consistent with disseminated intravascular coagulation in a rat model. J Infect Dis 2013, 208: 978-989

38. Jouvion G, Corre JP, Khun H, Moya-Nilges M, Roux P, Latroche C, Tournier JN, Huerre M, Chrétien F, Goossens PL: Physical sequestration of Bacillus anthracis in the pulmonary capillaries in terminal infection. J Infect Dis 2016, 214:281-287

39. Shah AV, Birdsey GM, Randi AM: Regulation of endothelial homeostasis, vascular development and angiogenesis by the transcription factor ERG. Vascul Pharmacol 2016, 86:3-13

40. Yuan L, Nikolova-Krstevski V, Zhan Y, Kondo M, Bhasin M, Varghese L, Yano K, Carman CV, Aird WC, Oettgen P: Antiinflammatory effects of the ETS factor ERG in endothelial cells are mediated through transcriptional repression of the interleukin- 8 gene. Circ Res 2009, 104:1049-1057

41. Yuan L, Le Bras A, Sacharidou A, Itagaki K, Zhan Y, Kondo M, Carman CV, Davis GE, Aird WC, Oettgen P: ETS-related gene (ERG) controls endothelial cell permeability via transcriptional regulation of the claudin 5 (CLDN5) gene. J Biol Chem 2012, 287:6582-6591

42. Liew PX, Kubes P: The neutrophil's role during health and disease. Physiol Rev 2019, 99:1223-1248

43. Papayannopoulos V: Neutrophil extracellular traps in immunity and disease. Nat Rev Immunol 2018, 18:134-147

44. Metzemaekers M, Gouwy M, Proost P: Neutrophil chemoattractant receptors in health and disease: double-edged swords. Cell Mol Immunol 2020, 17:433-450
45. Liu S, Miller-Randolph S, Crown D, Moayeri M, Sastalla I, Okugawa S, Leppla SH: Anthrax toxin targeting of myeloid cells through the CMG2 receptor is essential for establishment of $\mathrm{Ba}$ cillus anthracis infections in mice. Cell Host Microbe 2010, 8: 455-462

46. Liu JZ, Ali SR, Bier E, Nizet V: Innate immune interactions between Bacillus anthracis and host neutrophils. Front Cell Infect Microbiol 2018, 8:2

47. Karasu E, Nilsson B, Köhl J, Lambris JD, Huber-Lang M: Targeting complement pathways in polytrauma- and sepsis-induced multipleorgan dysfunction. Front Immunol 2019, 10:543

48. Wood AJT, Vassallo A, Summers C, Chilvers ER, Conway-Morris A: C5a anaphylatoxin and its role in critical illness-induced organ dysfunction. Eur J Clin Invest 2018, 48:e13028

49. Ward PA: The dark side of C5a in sepsis. Nat Rev Immunol 2004, 4: 133-142

50. Haviland DL, McCoy RL, Whitehead WT, Akama H, Molmenti EP, Brown A, Haviland JC, Parks WC, Perlmutter DH, Wetsel RA: Cellular expression of the C5a anaphylatoxin receptor (C5aR): demonstration of $\mathrm{C} 5 \mathrm{aR}$ on nonmyeloid cells of the liver and lung. J Immunol 1995, 154:1861-1869

51. Yuen J, Pluthero FG, Douda DN, Riedl M, Cherry A, Ulanova M, Kahr WHA, Palaniyar N, Licht C: NETosing neutrophils activate complement both on their own NETs and bacteria via alternative and non-alternative pathways. Front Immunol 2016, 7:137

52. Keragala CB, Draxler DF, McQuilten ZK, Medcalf RL: Haemostasis and innate immunity - a complementary relationship: a review of the intricate relationship between coagulation and complement pathways. Br J Haematol 2018, 180:782-798

53. Lupu F, Keshari RS, Lambris JD, Coggeshall KM: Crosstalk between the coagulation and complement systems in sepsis. Thromb Res 2014, 133(Suppl 1):S28-S31

54. Skjeflo EW, Christiansen D, Fure H, Ludviksen JK, Woodruff TM, Espevik T, Nielsen EW, Brekke OL, Mollnes TE: Staphylococcus aureus-induced complement activation promotes tissue factor-mediated coagulation. J Thromb Haemost 2018, 16: 905-918

55. Müller-Redetzky H, Kellermann U, Wienhold SM, Gutbier B, Lienau J, Hellwig K, Reppe K, Letsiou E, Tschernig T, Scholz M, Ahnert P, Maasch C, Hoehlig K, Klussmann S, Vater A, Firsching TC, Hoppe J, Suttorp N, Witzenrath M: Neutralizing complement $\mathrm{C} 5$ a protects mice with pneumococcal pulmonary sepsis. Anesthesiology 2020, 132:795-807

56. Grover SP, Mackman N: Tissue factor: an essential mediator of hemostasis and trigger of thrombosis. Arterioscler Thromb Vasc Biol 2018, 38:709-725

57. Bastarache JA, Wang L, Geiser T, Wang Z, Albertine KH, Matthay MA, Ware LB: The alveolar epithelium can initiate the extrinsic coagulation cascade through expression of tissue factor. Thorax 2007, 62:608-616 